\title{
FlORA y VEGETACIÓN DE LA SIERRA El RINCÓN, QuerÉTARO Y Michoacán, MÉXICO
}

\author{
José Alejandro Cabrera-Luna ${ }^{1,3}$, Hilda Edith Huerta-Cantera, Patricia Salinas-Soto 2 \\ y Diana Olvera-Valerio \\ 'Herbario "Dr. Jerzy Rzedowski", Facultad de Ciencias Naturales, Universidad Autónoma de Querétaro, \\ Campus Juriquilla. Querétaro, Querétaro, México \\ ${ }^{2}$ Secretaría de Desarrollo Agropecuario, Querétaro, Querétaro, México. \\ ${ }^{3}$ Autor para la correspondencia: jose.alejandro.cabrera@uaq.mx
}

\begin{abstract}
Resumen: Se analizó la riqueza florística, el hábito y la forma de vida, la presencia de especies nativas e introducidas, la flora sinantrópica, los patrones de distribución y endemismo, el estado de conservación de las plantas vasculares y se caracterizó los tipos de vegetación presentes en la Sierra El Rincón, Querétaro y Michoacán, México. Se registraron 79 familias, 216 géneros, 333 especies y 23 taxa infraespecíficos. Las familias con la mayor riqueza florística fueron Asteraceae (67), Poaceae (28) y Fabaceae (13). En cuanto a los géneros destacaron Quercus y Stevia. El hábito herbáceo está presente en el $84.1 \%$ de las especies y las formas de vida predominantes fueron las hemicriptófitas $(45.1 \%)$ y terófitas $(12.9 \%)$. El $96 \%$ de la flora es nativa a México y el $36.6 \%$ son indicadoras de disturbio. Se registraron siete patrones de distribución geográfica, donde se destaca que el 35.4 \% (118) de la flora es endémica de México. Así como dos patrones de endemismo, en los cuales 29 especies (8.7 \%) limitan su distribución al centro del país y 89 (26.7 \%) presentan una amplia distribución en el territorio nacional. De acuerdo con la NOM-059-SEMARNAT-2010 son Especies Sujetas a Protección Especial a Gentiana spathacea y Mammillaria rhodantha subsp. pringlei, y como Amenazada se encuentra Dasylirion acrotrichum. Se reconocieron siete tipos de vegetación, de los cuales el bosque de Quercus y de QuercusPinus son los tipos dominantes en el área de estudio. Además de contribuir significativamente a la riqueza florística de la región, esta sierra brinda diversos servicios ambientales a la población como la recarga de acuíferos, flora útil y áreas de esparcimiento. Es necesario coordinar esfuerzos y desarrollar un plan de manejo integral dirigido a la conservación y al uso sustentable de la flora y vegetación de la Sierra El Rincón.
\end{abstract}

Palabras Clave: Amealco, bosque de encino-pino, Epitacio Huerta, Flora del Bajío, Huimilpan.

\begin{abstract}
In this work the taxonomic richness, habit and life form, presence of native and introduced species, sinantropic flora, distribution and endemism patterns, and species conservation status were analyzed, and the type of vegetation was characterized in Sierra El Rincón, Querétaro and Michoacán states, Mexico. Seventy nine families, 216 genera, 333 species, and 23 infraspecific taxa were recorded. Asteraceae (67), Poaceae (28) and Fabaceae (13) were the richest families. For genera, Quercus and Stevia were the richest. The herbaceous habit is present in $84.1 \%$ of species and the main life forms were hemicryptophytes (45.1\%) and therophytes $(12.9 \%)$. From total flora, $96 \%$ are native to Mexico and $36.6 \%$ are disturbance indicators. Seven geographic distribution patterns were recorded, from total flora, $35.4 \%$ (118) is endemic to Mexico. Two endemism patterns were recorded, in which 29 species $(8.7 \%$ ) have their distribution limited to center of Mexico, and 89 species $(26.7 \%)$ show a wide distribution in Mexico. According to NOM-059-SEMARNAT-2010, Gentiana spathacea and Mammillaria rhodantha subsp. pringlei are considered as species in Special Protection and Dasylirion acrotrichum as a Threated specie. Seven types of vegetation were recognized, Quercus and Quercus-Pinus forests were dominant in the studied area. Besides significant contribution to floristic richness to this region, this sierra provides several environmental services like aquifers recharging, useful flora, and recreation areas. Development of an integral management plan for conservation and sustainable use of flora and vegetation of Sierra El Rincon is needed.
\end{abstract}

Key words: Amealco, Epitacio Huerta, Flora of Bajio, Huimilpan, oak-pine forest.

$\mathbf{L}$ a diversidad biológica de México es ampliamente reconocida por el número de especies de flora y fauna, razón por la que se le considera como un país megadiverso (Mittermeier et al., 1997). Esta riqueza es producto de su ubica- ción geográfica, su accidentada fisiografía, la presencia de diversas condiciones climáticas y su compleja historia geológica (Rzedowski, 1978; Ferrusquía-Villafranca, 1993). Todos estos factores dan como resultado un mosaico diverso 
de comunidades vegetales (Rzedowski, 1978), de las cuales las templadas y xerófilas destacan por su riqueza florística, la alta presencia de especies endémicas, sus variadas formas de vida y su amplia distribución (Rzedowski, 1978; Rzedowski 1991a; Rzedowski 1991b; Villaseñor y Ortiz, 2014).

Los bosques templados en México, son comunidades vegetales distribuidas principalmente en los sistemas montañosos, entre los 2,000 y 4,000 m, en climas templados y subhúmedos. Estas comunidades están dominadas fisionómicamente por especies de coníferas y de encinos o una combinación de ambos (Miranda y Hernández X., 1963; Rzedowski, 1978). Villaseñor y Ortiz (2014) estimaron su riqueza en 8,824 especies, de las cuales cerca del $50 \%$ $(4,534)$ son endémicas de México. Aunado a su valor biológico, se suma la importancia económica que poseen varias de sus especies vegetales, las cuales han sido objeto de explotación racional e irracional (Rzedowski, 1978; Espejel et al., 1999; Luna et al., 2003).

Los bosques templados mexicanos presentan una tasa de deforestación considerable (Toledo y Ordoñez, 1993; Challenger, 1998; Aguilar et al., 2000; Velázquez et al., 2002; INEGI, 2003; INEGI, 2005; Sánchez et al., 2009). De acuerdo con los datos presentados por el INEGI (2003; 2005), en el año 2003 estos bosques ocupaban 43.96 millones de hectáreas, para el 2005, se calculaba que se había perdido cerca del $27 \%$ de la superficie ocupada en años anteriores. En la actualidad estas cifras pueden ser aún más alarmantes. Ejemplo de ello, son los bosques templados del centro del país, los cuales han sido modificados y utilizados para la obtención de combustible (leña y carbón); de celulosa para la elaboración de papel; de madera para la construcción de muebles, viviendas y herramientas; e indirectamente en la obtención de hojarasca, humus (comercializados en los mercados como "tierra para macetas"), y de especies con valor ornamental (Cabrera-Luna et al., 2007) y medicinal. En algunos casos, han sido talados y sustituidos por zonas urbanas, agrícolas, ganaderas o por plantaciones de Eucalyptus, quedando los bosques fragmentados, vulnerables y aislados en las partes altas de las montañas (Zamudio et al., 1992). Lo antes mencionado se observa en la Sierra El Rincón, localizada entre los estados de Querétaro y Michoacán. Esta sierra alberga un manchón continuo y de extensión considerable de bosque de encino y de encino-pino (Zamudio et al., 1992), rodeado por zonas agrícolas y ganaderas, matorrales y pastizales secundarios, zonas reforestadas y pequeñas áreas sin vegetación donde se presenta una fuerte erosión.

A pesar de los esfuerzos que se han desarrollado en las últimas décadas por conocer e inventariar la flora de varias regiones de México, entre ellos el proyecto "Flora del Bajío y de Regiones Adyacentes", el cual abarca los estados de Guanajuato, Querétaro y norte de Michoacán (Calderón de Rzedowski y Rzedowski, 1991), todavía existen áreas en las que el conocimiento sobre sus recursos vegetales es incompleto (Llorente-Bousquets y Ocegueda, 2008). Dichos es- fuerzos se ven superados por la deforestación y destrucción del hábitat, lo que nos lleva a la pérdida de la biodiversidad (Dirzo, 1990; Dirzo y Gómez, 1996; Ceballos y OrtegaBaes, 2011; Martínez-Meyer et al., 2014).

Para la Sierra El Rincón no existe un conocimiento florístico previo que nos indique su importancia biológica. Por lo anterior y lo vulnerable a la pérdida de la biodiversidad de la Sierra El Rincón, se planteó estudiar su flora y vegetación, para generar un inventario florístico, caracterizar los tipos de vegetación y sentar las bases para la conservación y manejo de sus recursos vegetales, además de contribuir al conocimiento biológico de la región.

\section{Materiales y Métodos}

Área de estudio. La Sierra El Rincón se ubica entre los municipios de Huimilpan y Amealco, en el estado de Querétaro, y una pequeña porción se localiza en el municipio de Epitacio Huerta en el estado de Michoacán. Sus coordenadas extremas son: $\mathrm{X}=368573, \mathrm{Y}=2246981$ al norte $\mathrm{y} \mathrm{X}=368179$, $\mathrm{Y}=$ 2239471 al sur (Figura 1). La altitud varía de los 2,450 a los 2,850 m, siendo el punto más alto el cerro La Sanguijuela, en Amealco, Querétaro. Esta sierra pertenece a la región conocida como Sierra Queretana y a la provincia fisiográfica de la Faja Volcánica Transmexicana (García-Tuena et al., 2005), y su superficie estimada es de 5,520 ha.

El clima predominante es templado subhúmedo, con una precipitación anual de 700 a $800 \mathrm{~mm}$. El tipo de suelo es Luvisol rojizo. La vegetación corresponde a bosques Quercus, Quercus-Pinus, Pinus, matorral y pastizal (Zamudio et al., 1992), y en las partes bajas se observan zonas de cultivo de maíz y frijol.

Trabajo de campo y gabinete. De octubre de 2010 a diciembre de 2013, se hicieron colectas de ejemplares botánicos a través de recorridos periódicos en el área de estudio. El material vegetal colectado se procesó mediante las técnicas convencionales para cada grupo taxonómico (Lot y Chiang, 1986) y se determinó con ayuda de los siguientes tratamientos: Flora del Bajío y de regiones adyacentes (Calderón de Rzedowski y Rzedowski, 1991), Flora Neotropica (Farjon y Styles, 1997), Flora fanerogámica del valle de México (Calderón de Rzedowski y Rzedowski, 2001) y The pteridophytes of Mexico (Mickel y Smith, 2004); y algunos ejemplares fueron revisados por especialistas. La colección principal de los ejemplares botánicos se encuentra depositada en el herbario "Dr. Jerzy Rzedowski (QMEX)" de la Universidad Autónoma de Querétaro, y los duplicados serán distribuidos a otros herbarios nacionales. La vegetación de la Sierra El Rincón se caracterizó en base a observación directa siguiendo los criterios de Zamudio et al. (1992).

Se analizó la riqueza taxonómica, el hábito y la forma de vida, la presencia de especies nativas e introducidas, la flora sinantrópica, los patrones de distribución y endemismo, además del estado de conservación de las especies vegeta- 


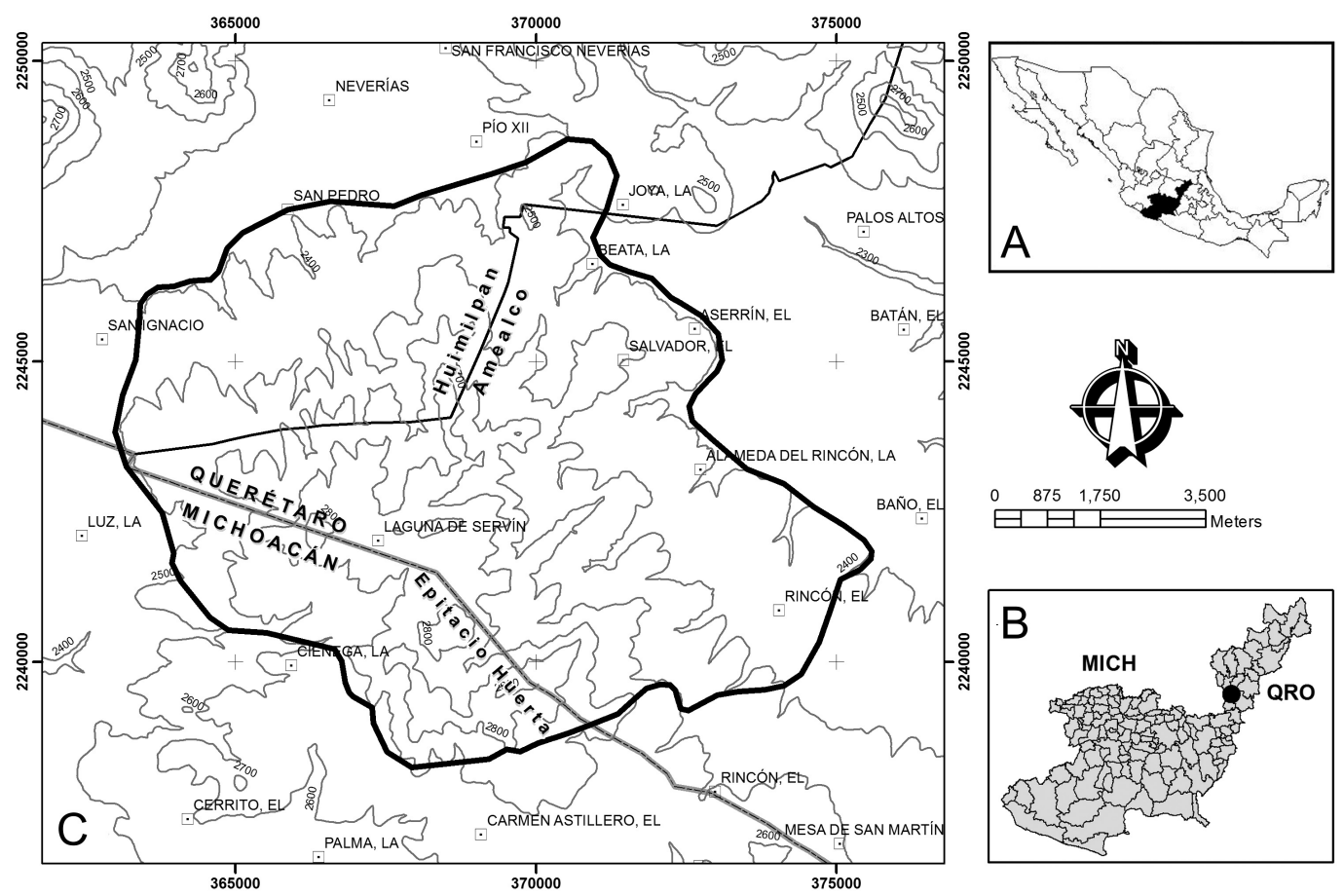

Figura 1. Localización del área de estudio. A) Ubicación geográfica de los estados de Michoacán y Querétaro. B) Localización de la Sierra El Rincón en Michoacán (MICH) y Querétaro (QRO). C) Sierra El Rincón, mostrando cotas altitudinales, principales poblados y límites municipales y estatales.

les presentes en la Sierra El Rincón. Con esta información se elaboró un listado florístico (Apéndice), siguiendo las propuestas de clasificación de Mickel y Smith (2004) para Pteridophyta y grupos afines; Farjon (2001) para Pinophyta y los del APG III (2009) para angiospermas. Los nombres científicos se citan de acuerdo con el Índice Internacional de Nombres de Plantas (IPNI por sus siglas en inglés) <www. ipni.org $>$ (consulta mayo 2014) y a la base de datos del Missouri Botanical Garden $\mathrm{W}^{3}$ Tropicos <www.tropicos.org> (consulta mayo 2014).

Los hábitos y las formas de vida se asignaron por observación directa durante la colecta, siguiendo los criterios de Judd et al. (2002) para el hábito y el sistema propuesto por Raunkiaer (1934), y modificado por Mueller-Dombois y Ellenberg (1974) para las formas de vida. Las especies introducidas y la flora sinantrópica se reconocieron al consultar las siguientes obras: Villaseñor y Espinosa-García (1998), Calderón de Rzedowski y Rzedowski (2001), Suárez-Ramos et al. (2004), Villaseñor y Espinosa-García (2004), y el sitio web Malezas de México < www.conabio.gob.mx> (consulta abril 2014).

Los patrones de distribución y de endemismo se establecieron para cada especie a partir de la literatura empleada en la determinación taxonómica. Las especies se agruparon en los siguientes patrones de distribución geográfica: distribución mundial, amplia en América, de México a Sudamérica, de México a Centroamérica, de México a Norteamérica, México y las Antillas, y endémica de México. Para el ende- mismo, se agruparon en los siguientes patrones: con amplia distribución en México y, endémica del centro de México (Guanajuato, Querétaro, norte de Michoacán, Hidalgo, Estado de México, Distrito Federal, Tlaxcala, Morelos y Puebla). El estado de conservación de cada una de las especies se obtuvo de la Norma Oficial Mexicana NOM-059-SEMARNAT-2010 (SEMARNAT, 2010).

\section{Resultados}

Inventarío florístico. La flora de la Sierra El Rincón está representada por 79 familias, 216 géneros, 333 especies, nueve subespecies y 14 variedades (Apéndice). El grupo que presentó la mayor riqueza fue Eudicotyledoneae, seguido de Monocotyledoneae, Pteridophyta, Pinophyta y Magnoliidae (Tabla 1). La familia mejor representada fue Asteraceae con 36 géneros y 67 especies, seguida de Poaceae (22/28),

Tabla 1. Riqueza de la flora vascular presente en la Sierra El Rincón.

\begin{tabular}{lccccc}
\hline GRUPO & Familia & Género & Especie & Subespecie & Variedad \\
\hline Eudicotyledoneae & 53 & 146 & 229 & 7 & 10 \\
Monocotyledoneae & 14 & 50 & 71 & - & 2 \\
Pteridophyta & 9 & 17 & 25 & 2 & 2 \\
Pinophyta & 2 & 2 & 7 & - & - \\
Magnoliidae & 1 & 1 & 1 & - & - \\
TOTAL & 79 & 216 & 333 & 9 & 14 \\
\hline
\end{tabular}


José Alejandro Cabrera-Luna et AL.

Tabla 2. Riqueza de especies y géneros por familias presentes en la Sierra El Rincón.

\begin{tabular}{lcccc}
\hline Familia & $\begin{array}{c}\text { Número de } \\
\text { especies }\end{array}$ & Porcentaje & $\begin{array}{c}\text { Número de } \\
\text { géneros }\end{array}$ & Porcentaje \\
\hline Otras familias (63) & 124 & 37.3 & 99 & 45.8 \\
Asteraceae & 67 & 20.1 & 36 & 16.7 \\
Poaceae & 28 & 8.4 & 22 & 10.2 \\
Fabaceae & 13 & 3.9 & 10 & 4.6 \\
Lamiaceae & 11 & 3.3 & 5 & 2.3 \\
Pteridaceae & 11 & 3.3 & 5 & 2.3 \\
Cyperaceae & 10 & 3 & 5 & 2.3 \\
Fagaceae & 9 & 2.7 & 1 & 0.5 \\
Solanaceae & 8 & 2.4 & 6 & 2.8 \\
Caryophyllaceae & 7 & 2.1 & 6 & 2.8 \\
Apiaceae & 7 & 2.1 & 4 & 1.9 \\
Orobanchaceae & 7 & 2.1 & 3 & 1.4 \\
Asparagaceae & 7 & 2.1 & 6 & 2.8 \\
Onagraceae & 6 & 1.8 & 3 & 1.4 \\
Plantaginaceae & 6 & 1.8 & 3 & 1.4 \\
Juncaceae & 6 & 1.8 & 1 & 0.4 \\
Pinaceae & 6 & 1.8 & 1 & 0.4 \\
TOTAL & 333 & 100 & 216 & 100 \\
\hline
\end{tabular}

Fabaceae (10/13), Lamiaceae (5/11), Pteridaceae (5/11) y Cyperaceae (5/10). En estas seis familias se encuentra representado el $38.1 \%$ (83) de los géneros y el $42 \%$ (140) de las especies. Las 73 familias restantes registraron menos de diez especies cada una (Tabla 2).

Los géneros que presentaron la mayor riqueza fueron Quercus y Stevia con nueve especies cada uno; seguidos de Juncus, Pinus y Salvia con seis, Muhlenbergia, Pseudognaphalium y Tagetes con cinco y Cyperus, Desmodium, Oenothera y Roldana con cuatro. Estos 12 géneros albergaron el 20.7 \% (69) de las especies registradas en el área de estudio. El resto de los géneros (204) presentaron tres o menos especies.

El hábito herbáceo está presente en 280 especies (84.1\%),

Tabla 3. Riqueza de las forma de vida* presentes en la Sierra El Rincón. * Raunkiaer (1934) y modificado por Mueller-Dombois y Ellenberg (1974).

\begin{tabular}{lcc}
\hline Formas de vida & Número de especies & Porcentaje \\
\hline Hemicriptófita & 150 & 45.1 \\
Terófita & 43 & 12.9 \\
Caméfita & 30 & 9 \\
Fanerófita cespitosa & 25 & 7.5 \\
Fanerófita escaposa & 25 & 7.5 \\
Hidrófita & 24 & 7.2 \\
Geófita & 19 & 5.7 \\
Liana & 9 & 2.7 \\
Parásita & 4 & 1.2 \\
Epífita & 4 & 1.2 \\
TOTAL & 333 & 100 \\
\hline
\end{tabular}

Tabla 4. Patrones de distribución geográfica de las especies presentes en la Sierra El Rincón.

\begin{tabular}{lcc}
\hline Distribución & Número de especies & Porcentaje \\
\hline Endémica de México & 118 & 35.4 \\
Amplia en América & 71 & 21.3 \\
de México a Centroamérica & 55 & 16.6 \\
Distribución mundial & 30 & 9 \\
de México a Sudamérica & 33 & 9.9 \\
de México a Norteamérica & 25 & 7.5 \\
México y las Antillas & 1 & 0.3 \\
TOTAL & 333 & 100 \\
\hline
\end{tabular}

seguido del arbustivo (28/8.4 \%) y el arbóreo (25/7.5\%). En cuanto a las formas de vida, las hemicriptófitas son la forma de vida mejor representada en la flora de la Sierra El Rincón. Le siguen en número de importancia las terófitas, caméfitas, hidrófitas, fanerófitas cespitosas, fanerófitas escaposas, geófitas, lianas, parásitas y epífitas (Tabla 3).

Del total de especies registradas, el $96 \%$ (320) son nativas de México y el $4 \%$ (13) son introducidas. La flora sinantrópica (malezas o indicadoras de disturbio) está representada por $122(36.6 \%)$ especies, de las cuales 109 son nativas y 13 son introducidas (Apéndice). En cuanto a los patrones de distribución geográfica (Tabla 4), cabe destacar que el $35.4 \%$ (118) de la flora de la Sierra El Rincón es endémica de México, seguido con el $21.3 \%$ (71) las especies con amplia distribución en América. Se identificaron dos patrones en las especies endémicas de México, en los cuales, $29(8.7 \%)$ limitan su distribución al centro del país y 89 (26.7 \%) presentan una amplia distribución en el territorio nacional. De acuerdo con la NOM-059-SEMARNAT-2010, dos especies están en la categoría de Especie Sujeta a Protección Especial (Gentiana spathacea y Mammillaria rhodantha subsp. pringlei) y una como Amenazada (Dasylirion acrotrichum).

Vegetación. En la Sierra El Rincón se reconocieron siete tipos de vegetación: bosque de Quercus, bosque de Quercus-Pinus, bosque de Pinus, matorral crasicaule, pastizal, vegetación acuática y vegetación subacuática. Siendo el bosque de Quercus y el bosque de Quercus-Pinus los tipos de vegetación dominantes en la Sierra El Rincón.

El bosque de Quercus se desarrolla entre los 2,450 y 2,800 m. Fisonómicamente es un bosque subcaducifolio con árboles de 5 a $15 \mathrm{~m}$ de altura como: Buddleja cordata, Crataegus mexicana, Prunus serotina subsp. capuli, Quercus castanea, $Q$. crassifolia, $Q$. crassipes, $Q$. eduardii, $Q$. laeta, $Q$. laurina y $Q$. rugosa. Entre los arbustos destacan: Arctostaphylos pungens, Baccharis pteronioides, Bouvardia ternifolia, Calliandra grandiflora, Monotropa hypopitys y Montanoa grandiflora. Las hierbas están representadas por: Begonia gracilis, Bletia neglecta, Echeveria mucronata, Helianthemum glomeratum, Lamourouxia dasyantha, Ma- 
mmillaria rhodantha subsp. pringlei, Muhlenbergia pubescens, M. robusta, Phytolacca icosandra, Pseudognaphalium purpurascens, Salvia elegans, Silene laciniata, Selaginella pallescens, Tagetes erecta y Tillandsia tortilis. Cladocolea diversifolia y Conopholis alpina se encontraron parasitando a diversas especies de Quercus, y Tillandsia juncea y T. recurvata creciendo como epífitas.

El bosque de Quercus-Pinus se desarrolla en un intervalo altitudinal de 2,500 a los 2,850 m, en lugares planos o con pendiente pronunciada. Fisonómicamente es un bosque perennifolio a subcaducifolio con individuos de 10 a $25 \mathrm{~m}$ de altura. En el estrato arbóreo están presentes especies como: Arbutus tessellata, A. xalapensis, Clethra mexicana, Pinus hartwegii, P. leiophylla, P. montezumae, P. patula, P. pseudostrobus, $P$. teocote, Quercus candicans, $Q$. castanea, $Q$. crassifolia, $Q$. crassipes, $Q$. laeta, $Q$. laurina, $Q$. obtusata y Q. rugosa. Entre los componentes arbustivos destacan: Berberis moranensis, Cestrum thyrsoideum, Cornus excelsa, Monnina ciliolata y Rhamnus microphylla. Entre las hierbas se distinguen: Cirsium pinetorum, Cosmos bipinnatus, Dahlia coccinea, Gentiana spathacea, Geranium bellum, Iostephane heterophylla, Macromeria longiflora, Malaxis fastigiata, M. soulei, Muhlenbergia pubescens, Packera sanguisorbae, Pteridium aquilinum var. feei, Ranunculus petiolaris var. arsenei, Salvia mexicana, S. patens, Schizachyrium sanguineum, Stevia elatior, S. eupatoria, S. origanoides, Trisetum virletii y Viola grahamii. Las epífitas y parásitas están representadas por: Arceuthobium vaginatum, Conopholis alpina, Elaphoglossum petiolatum, Phoradendron velutinum y Polypodium madrense, y las lianas por: Ipomoea madrensis y Smilax moranensis.

El bosque de Pinus se encuentra escasamente representado en la Sierra El Rincón, desarrollándose en lugares planos, a altitudes desde los 2,700 a 2,850 m, y rodeado de bosque de Quercus-Pinus. En él se encuentran árboles de P. montezumae de 20 a $25 \mathrm{~m}$ de altura. Los arbustos, las hierbas, epífitas, parásitas y lianas se encuentran pobremente representados.

El pastizal se localiza en claros del bosque de Quercus y del bosque de Quercus-Pinus entre los 2,450 y 2,800 m, en sitios planos o con pendiente poco pronunciada. Entre los elementos herbáceos destacan las gramíneas como: Aristida schiedeana, A. ternipes, Chloris submutica, Digitaria ternata, Hilaria belangeri, Poa annua y Sporobolus indicus. Dentro de esta vegetación se observan creciendo a Dasylirion acrotrichum.

El matorral crasicaule se desarrolla entre los 2,450 y $2,550 \mathrm{~m}$. Se localiza en lugares planos o en pendientes poco pronunciadas, y colindando con zonas de cultivo y asentamientos habitacionales. Se encuentran arbustos espinosos de 1 a $2 \mathrm{~m}$ de altura como: Mimosa biuncifera, Opuntia robusta, O. tomentosa y Painteria leptophylla. Entre las herbáceas se encuentran: Asclepias linaria, Astrolepis sinuata, Cheilanthes bonariensis, Loeselia caerulea, L. mexicana,
Muhlenbergia minutissima y Tragia nepetifolia. Los árboles, epífitas, parásitas y lianas se encuentran ausentes.

La vegetación acuática prospera en sitios con drenaje deficiente dentro del bosque de Quercus y del bosque de Quercus-Pinus donde se forman estanques temporales o permanentes, en pequeños bordos y en la presa de la comunidad La Beata, Amealco, Querétaro. Esta vegetación está conformada por herbáceas de 10 a $90 \mathrm{~cm}$ de alto, entre las que encontramos a: Acmella repens, Cyperus niger, Echinochloa oplismenoides, Eleocharis ignota, E. montevidensis, Eriocaulon benthamii, Equisetum hyemale subsp. affine, Heteranthera limosa, Juncus acuminatus, Limosella aquatica, Polygonum hydropiperoides, P. mexicanum, Ranunculus hydrocharoides, Rorippa mexicana, Utricularia perversa y Typha domingensis.

La vegetación subacuática se desarrolla a la orilla del arroyo de la comunidad La Beata, donde prosperan árboles de 7 a 15 m de altura de Alnus jorullensis subsp. jorullensis y Salix bonplandiana. En este tipo de vegetación se encuentran creciendo diversos helechos, entre los que destacan: Adiantum andicola, A. capillus-veneris, A. poiretii y Woodwardia spinulosa.

\section{Discusión}

La flora de la Sierra El Rincón representa el 6 \% (333 especies) de las 5,500 especies de plantas vasculares estimadas por Rzedowski (1997) para la Flora del Bajío y de Regiones Adyacentes, y cerca del $3.7 \%$ de las especies registradas por Villaseñor y Ortiz (2014) para los bosques templados mexicanos. Esta riqueza florística es significativa, ya que el área de estudio ocupa alrededor del $0.1 \%$ con respecto a la superficie que abarca el proyecto Flora del Bajío y de Regiones Adyacentes (aproximadamente 50,000 $\mathrm{Km}^{2}$ ) (Rzedowski, 1997). Aunado a lo anterior, esta riqueza florística es ligeramente superada por la de la Sierra de Santa Rosa, Guanajuato (496 especies), cuya superficie es cuatro veces mayor a la del área estudiada (Martínez-Cruz y TéllezValdés, 2004). Al comparar nuestros resultados con otros estudios florísticos llevados a cabo en Guanajuato (Quero, 1984), norte de Michoacán (Cornejo-Tenorio et al., 2003) y Querétaro (Fernández y Colmenero, 1997; Baltazar et al., 2004; Cabrera-Luna y Gómez-Sánchez, 2005), se observa que las cifras registradas en este trabajo son superiores a las reportadas en los estudios mencionados.

Según Villaseñor (2003) y Mickel y Smith (2004) algunas de las familias más ricas y diversas en la flora mexicana son: Asteraceae, Cyperaceae, Fabaceae, Lamiaceae, Poaceae y Pteridaceae; resultados que fueron corroborados en el presente trabajo. Los géneros registrados con mayor riqueza de especies en la Sierra El Rincón (Cyperus, Desmodium, Juncus, Muhlenbergia, Oenothera, Pinus, Pseudognaphalium, Quercus, Roldana, Salvia, Stevia y Tagetes), también se encuentran ricamente representados en México (Villaseñor, 
2004). Nuestro país es considerado como centro de diversidad de Pinus, mientras que para Quercus y Salvia, México está considerado como uno de los centro de diversidad, particularmente en las regiones montañosas y templadas (Rzedowski, 1991; Styles, 1993; Farjon y Styles, 1997; Nixon, 1998; Ramamoorthy y Elliott, 1998; Valencia, 2004), lo que explica su alta riqueza en el área de estudio. Resalta que la Sierra El Rincón tiene un número importante de especies de encinos (9) y pinos (6) con respecto a otros sistemas montañosos del centro de México (Fernández y Colmenero, 1997; Cerros-Tlatilpa y Espejo-Serna, 1998; Figueroa-Rangel y Olvera-Vargas, 2000; Enríquez et al., 2003; Martínez-Cruz y Téllez-Valdés, 2004; Rubio-Licona et al., 2011; Cornejo-Tenorio, et al., 2013). Tan sólo esta sierra alberga el $20 \%$ de las especies de Quercus mencionadas por Romero-Rangel et al. (2014) para la Flora del Bajío y de Regiones Adyacentes.

La predominancia del hábito herbáceo, así como la alta presencia de hemicriptófitas y terófitas en la Sierra El Rincón, es común en la flora de México, ya que las herbáceas son el grupo de plantas con la mayor riqueza y diversidad en el país (Villaseñor y Ortiz, 2014), sobre todo en las regiones montañosas y templadas (Rzedowski, 1978; Rzedowski y Calderón de Rzedowski, 1989). Otro factor que puede estar favoreciendo a la presencia de tan variadas formas de vida es la heterogeneidad ambiental en el área estudiada.

A pesar de los diversos agentes de perturbación observados en la sierra, ésta conserva un número importante de especies nativas (320), de las cuales, 109 forman parte de la flora sinantrópica de México, estas últimas reflejan la perturbación antrópica presente en la Sierra El Rincón. En cuanto a la flora introducida, la cual representa el $4 \%$, destacan dos especies por los posibles daños que pueden ocasionar a la flora nativa del lugar: Eucalyptus camaldulensis, árbol australiano utilizado para reforestación y como ornamental en México (Granados-Sánchez y López-Ríos, 2007), y que presenta alelopatía (Rice, 1979). Estos árboles se encuentran creciendo en los alrededores de algunas viviendas, a orillas de caminos y carreteras. Senecio inaequidens, es una hierba africana considerada como invasora agresiva, la cual puede estar compitiendo y desplazando a otras especies vegetales, principalmente herbáceas (Rzedowski et al., 2003). Ambas especies ameritan atención especial, para su control y disminución en número dentro del área de estudio, ya que pueden afectar la riqueza florística y por consiguiente la diversidad del lugar, principalmente de hierbas.

Entre los agentes de perturbación observados, sobresalen: (1) la tala ilegal, siendo más evidente esta actividad en la porción michoacana, es necesario y urgente llevar a cabo programas de reforestación y acciones para detener dicha actividad, permitiendo así la recuperación de la vegetación; (2) extracción de tierra y hojarasca, ambas afectan los ciclos biogeoquímicos, causando un empobrecimiento de nutrientes y del bancos de semillas en el suelo, afectando así a la dinámica poblacional de las especies vegetales; (3) extracción de cactáceas, crasuláceas, musgos, plántulas de pinos y líquenes, todos estos elementos son saqueados durante diciembre en diferentes grados de intensidad, con la finalidad de ser comercializados y utilizados en la decoración de hogares en la navidad, la educación y concientización ambiental son las herramientas más importantes que permitan frenar dicha actividad; (4) extracción de hongos comestibles como: Amanita caesarea, Cantharellus cibarius, Ramaria botrytis, $R$. flava y R. formosa, los cuales forman micorrizas con los encinos (García-Jiménez et al., 1998), son necesarios estudios más detallados que permitan conocer las afectaciones que causan la extracción de estos y otros hongos micorrízicos sobre el establecimiento de los encinos y (5) la apertura de áreas para la construcción de viviendas, de áreas de esparcimiento y de zonas de cultivo. Todos estos agentes causan la degradación del hábitat, la pérdida de especies, y el incremento en número y dispersión de las especies introducidas, y en su conjunto ponen en riesgo la riqueza y biodiversidad florística y faunística de la Sierra El Rincón.

Rzedowski (1978) considera a los elementos endémicos y a los meridionales como los más importantes para los bosques templados mexicanos y los resultados de este trabajo en la Sierra de El Rincón lo confirman. En cuanto a los patrones de endemismo observados, sobresalen las especies endémicas de México con amplia distribución, patrón comúnmente registrado en la flora mexicana (Rzedowski, 1991b).

De las especies registradas en la Norma Oficial Mexicana NOM-059-SEMARNAT-2010, cabe mencionar lo siguiente: la noche buena azul o flor de hielo (Gentiana spathacea), es una hierba endémica a México y está registrada en la NOM-059-SEMARNAT-2010 en la categoría de Especie Sujeta a Protección Especial, se distribuye en los bosques templados de los sistemas montañosos del centro y norte del país, en el Bajío se le encuentra creciendo en los bosques de pino-encino y pino-oyamel (Villarreal, 1998) y es utilizada como planta medicinal en el municipio de Amealco, Querétaro (Serrano et al., 1992). En el área de estudio está bien representada, sobre todo en la porción queretana de la sierra. Presenta un potencial ornamental considerable, por lo que su cultivo y propagación puede justificarse.

La biznaga dorada (Mammillaria rhodantha subsp. pringlei), es una cactácea endémica del centro de México (Scheinvar, 2005) y en la NOM-059-SEMARNAT-2010 se encuentra en la categoría de Especie Sujeta a Protección Especial. Esta especie es extraída de su hábitat y comercializada como ornamental en las festividades decembrinas (Cabrera-Luna et al., 2007), actividad que puede poner en riesgo su supervivencia. En el área de estudio se encontró una pequeña población de 30 individuos creciendo sobre rocas cubiertas con musgo dentro del bosque de encino-pino. Por lo que es necesario llevar a cabo estudios más detalla- 
dos sobre su biología que permitan desarrollar programas de propagación y reintroducción de la especie.

La cucharilla o sotol (Dasylirion acrotrichum), es una especie endémica del centro de México (Galván, 2001) que está registrada en la NOM-059-SEMARNAT-2010 como Amenazada. Esta especie es cada vez es más escasa en su hábitat, debido a la extracción de plantas femeninas con fines ornamentales y para la elaboración de adornos utilizados en fiestas religiosas locales, tradición que data desde tiempos prehispánicos (Haeckel, 2008). En la sierra El Rincón sólo se localizaron siete plantas femeninas y tres masculinas, las cuales estaban creciendo en lugares abiertos del bosque de pino-encino. La supervivencia de esta especie en el área de estudio es altamente crítica. Al igual que la biznaga dorada, es necesario conocer más sobre su biología para tener las herramientas y el conocimiento necesario para su conservación, propagación y reintroducción.

El bosque de Quercus y el bosque de Quercus-Pinus son los principales tipos de vegetación presentes en la Sierra El Rincón, le siguen el pastizal y el matorral crasicaule, los cuales al parecer son de origen secundario. El bosque de Pinus, la vegetación acuática y la subacuática, se encuentran poco representadas, no obstante aportan un número significativo de especies a la flora de la Sierra El Rincón. Son necesarios estudios más detallados sobre la vegetación presente en esta serranía. La Sierra El Rincón además de contribuir con una riqueza considerable a la flora de Querétaro y Michoacán, es un sistema montañoso que brinda servicios ambientales como la recarga de acuíferos, la conservación de suelos, la reducción de contaminación por dióxido de carbono. Así como áreas de esparcimiento para la población y ofrecer un número importante de especies útiles, las cuales ameritan ser estudiadas. Estos valores hacen de la Sierra El Rincón un ecosistema que se debe de conservar, ya que representa una área importante para que se desarrolle un plan específico para su restauración y aprovechamiento racional de sus recursos naturales. Este tipo de acciones resultan imperativas para evitar la pérdida de un ecosistema de singular valor paisajístico y biológico y que alberga uno de los dos bosques templados más importantes para los municipios de Amealco y Huimilpan (sur del estado de Querétaro).

\section{Agradecimientos}

Agradecemos a Fabiola Magallán Hernández, Raquel Galván Villanueva, Ana Rosa López Ferrari, Socorro González Elizondo, Adolfo Espejo Serna, José Luis Villaseñor, Eleazar Carranza, Luis Hernández Sandoval, Sergio Zamudio y Thomas F. Daniel, por la revisión de ejemplares de los grupos de los que son especialistas. También a Ma. del Pueblito Luna S., Andrea Cabrera L. y a Melisa Cabrera por su apoyo en campo, a Luis Ibarra por la elaboración del abstract y a Kathia Téllez por las sugerencias al manuscrito. Muy especialmente agradecemos al Sr. Alejandro Cabrera Z. $\dagger$ por su compañía durante las colectas, así como a los revisores por sus comentarios para la mejora de este manuscrito.

\section{Literatura citada}

Aguilar C., Martínez E. y Arriaga L. 2000. Deforestación y fragmentación del ecosistema: ¿Qué tan grave es el problema en México? Biodiversitas 30:7-11.

APG III. 2009. An update of the Angiosperm Phylogeny Group classification for the orders and families of flowering plants: APG III. Botanical Journal of the Linnean Society 161:105121.

Baltazar J., Martínez y Díaz M. y Hernández L. 2004. Guía de Plantas Comunes del Parque Nacional El Cimatario y sus Alrededores. Universidad Autónoma de Querétaro,

Querétaro.

Cabrera-Luna J.A. y Gómez-Sánchez M. 2005. Análisis florístico de La Cañada, México. Boletín de la Sociedad Botánica de México 77:35-50.

Cabrera-Luna J.A., Serrano-Cárdenas V. y Pelz-Marín R. 2007. Plantas vasculares comercializadas como ornamentales decembrinas en 12 municipios de Querétaro, México. Polibotánica 24:117-138.

Calderón de Rzedowski G. y Rzedowski J. 2001. Flora Fanerogámica del Valle de México. Instituto de Ecología y Comisión Nacional para el Conocimiento y Uso de la Biodiversidad, Pátzcuaro.

Ceballos G. y Ortega-Baes P. 2011. La sexta extinción: la pérdida de especies y poblaciones en el Neotrópico. En: Simonetti J. y Dirzo R. Eds. Conservación Biológica: Perspectivas de Latinoamérica, pp. 95-108, Editorial Universitaria, Santiago de Chile.

Cerros-Tlatilpa R. y Espejo-Serna A. 1998. Contribución al conocimiento florístico de los cerros El Sombrerito y Las Mariposas (Zoapapalotl) en el municipio de Tlayacapan, Morelos, México. Polibotánica 8:29-44.

Challenger A. 1998. Utilización y Conservación de los Ecosistemas Terrestres de México. Comisión Nacional para el Conocimiento y Uso de la Biodiversidad, México.

Cornejo-Tenorio G., Casas A., Farfán B., Villaseñor J.L. e IbarraManríquez G. 2003. Flora y vegetación de la zona núcleo de la reserva de la biosfera mariposa monarca, México. Boletín de la Sociedad Botánica de México 73:43-62.

Cornejo-Tenorio G., Sánchez-García E., Flores-Tolentino M., Santana-Michel F.J. e Ibarra-Manríquez E. 2013. Flora y vegetación del cerro El Águila, Michoacán, México. Botanical Sciences 91:155-180.

Dirzo R. 1990. La biodiversidad como crisis ecológica actual ¿qué sabemos? Ciencias 4:48-55.

Dirzo R. y Gómez G. 1996. Ritmos temporales de la investigación taxonómica de plantas vasculares en México y una estimación del número de especies conocidas. Annals of the Missouri Botanical Garden 83:396-403.

Enríquez E.D, Koch S.D. y González-Elizondo M.S. 2003. Flora y vegetación de la Sierra de Órganos, municipio de Sombrerete, Zacatecas, México. Acta Botanica Mexicana 64:45-89.

Espejel M.M., Santacruz N. y Sánchez M. 1999. El uso de los encinos en la región de La Malinche, estado de Tlaxcala, México. Boletín de la Sociedad Botánica de México 64:35-39. 
Farjon A. 2001. World Checklist and Bibliography of Conifers. The Royal Botanic Gardens, Kew, Londres.

Farjon A. y Styles B.T. 1997. Pinus (Pinaceae). Flora Neotropica. The New York Botanical Garden, Nueva York.

Fernández F. y Colmenero J.L. 1997. Notas sobre la vegetación y flora de San Joaquín, Querétaro, México. Polibotánica 4:10-36.

Ferrusquía-Villafranca I. 1993. Geology of México: a sinopsis. En: Ramamoorthy T. P., Bye R., Lot A. y Fa J. Eds. Biological Diversity of Mexico: Origins and Distribution, pp. 3-108, Oxford University Press, Nueva York.

Figueroa-Rangel B.L. y Olvera-Vargas M. 2000. Dinámica de la composición de especies en bosques de Quercus crassipes $\mathrm{H}$. et B. en Cerro Grande, Sierra de Manantlán, México. Agrociencia 34:91-98.

Galván R. 2001. Nolinaceae. En: Calderón de Rzedowski G. y Rzedowski J. Eds. Flora fanerogámica del Valle de México, pp. 1239-1240, Instituto de Ecología y Comisión Nacional para el Conocimiento y Uso de la Biodiversidad, Pátzcuaro.

García-Jiménez J., Pedraza D., Silva I., Andrade R.L. y Castillo J. 1998. Los Hongos del Estado de Querétaro, Universidad Autónoma de Querétaro, Querétaro.

Gómez-Tuena A., Orozco-Esquivel. M.T. y Ferrari. 2005. Petrogénesis ígnea de la Faja Volcánica Transmexicana. Boletín de la Sociedad Geológica Mexicana 42:227-283.

Granados-Sánchez D. y López-Ríos, G.F. 2007. Fitogeografía y ecología del género Eucalyptus. Revista Chapingo. Serie Forestal y del Ambiente 156: 143-156.

Haeckel I. 2008. The "arco floral": ethnobotany of Tillandsia and Dasylirion spp. in a Mexican religious adornment. Economic Botany 62:90-95.

INEGI. 2003. Conjunto de datos vectoriales de la carta de vegetación primaria, escala 1:1 000 000. Instituto Nacional de Estadística y Geografía, Aguascalientes.

INEGI. 2005. Conjunto de datos vectoriales de uso de suelo y vegetación. Serie III (continuo nacional), escala 1:250 000. Instituto Nacional de Estadística y Geografía, Aguascalientes.

International Plant Names Index. <www.ipni.org> (consulta marzo-abril 2014).

Judd W.S., Campbell C.S., Kellogg E.A., Stevens P.F. y Donoghue M.J. 2002. Plant Systematics: A Phylogenetic Approach. Sinauer, Sunderland.

Llorente-Bousquets J. y Ocegueda S. 2008. Estado del conocimiento de la biota. En: Capital Natural de México, vol. I: Conocimiento Actual de la Biodiversidad, pp. 283-322, Comisión Nacional para el Conocimiento y Uso de la Biodiversidad, México, D.F.

Lot A. y Chiang F. 1986. Manual de Herbario: Administración y Manejo de Colecciones, Técnicas de Recolección y Preparación de Ejemplares Botánicos. Consejo Nacional de la Flora de México A. C., México, D.F.

Luna A., Montalvo L. y Rendón B. 2003. Los usos no leñosos de los encinos. Boletín de la Sociedad Botánica de México 72:107-117.

Malezas de México <www.conabio.gob.mx> (consulta abril 2014).

Martínez-Cruz J. y Téllez-Valdés O. 2004. Listado florístico de la Sierra de Santa Rosa, Guanajuato, México. Boletín de la Sociedad Botánica de México 74:31-49.

Martínez-Meyer E., Sosa-Escalante J.E. y Álvarez F. 2014. El estudio de la biodiversidad en México: ¿una ruta con dirección? Revista Mexicana de Biodiversidad 85: 1-9.

Mickel J.T. y Smith A.R. 2004. The Pteridophytes of Mexico.
Vol. 88. Memoirs of the New York Botanical Garden, Nueva York.

Miranda F. y Hernández X. E. 1963. Los tipos de vegetación de México y su clasificación. Boletín de la Sociedad Botánica de México 28:29-179.

Missouri Botanical Garden $\mathrm{W}^{3}$ Tropicos. <www.mobot.org> (consulta marzo-abril 2014).

Mittermeier R., Goettsch C. y Robles-Gil P. 1997. Megadiversidad, los Países Biológicamente más Ricos del Mundo. CEMEX-Agrupación Sierra Madre, México, D.F.

Mueller-Dombois D. y Ellenberg H. 1974. Aims and Methods of Vegetation Ecology. John Wiley and Sons, Nueva York.

Nixon K. 1993. The genus Quercus in Mexico. En: Ramamoorthy T. P., Bye R., Lot A. y Fa J. Eds. Biological Diversity of Mexico: Origins and Distribution, pp. 447-458, Oxford University Press, Nueva York.

Ramamoorthy T.P. y Elliott M. 1993. Mexican Lamiaceae: diversity, distribution, endemism and evolution. En: Ramamoorthy, T. P., Bye R., Lot A. y Fa J. Eds. Biological Diversity of Mexico: Origins and Distribution, pp. 513-539, Oxford University Press, Nueva York.

Raunkiaer C. 1934. The Life Forms of Plants and Statistical Plant Geography. Clarendon Press, Oxford.

Rice E.L. 1979. Allelopathy-an update. The Botanical Review 45:17-109.

Romero-Rangel S., Rojas-Zenteno E.C. y Rubio-Licona L.E. 2014. Fagaceae. Fascículo 181. Flora del Bajio y de Regiones Adyacentes. Instituto de Ecología A. C. Centro Regional del Bajío, Pátzcuaro.

Rubio-Licona L.E., Romero-Rangel S. y Rojas-Zenteno E.C. 2011. Estructura y composición florística de dos comunidades con presencia de Quercus (Fagaceae) en el estado de México. Revista Chapingo Serie Ciencias Forestales y del Ambiente 17:77-90.

Rzedowski J. 1978. Vegetación de México. Limusa. México, D.F.

Rzedowski J. 1991a. Diversidad y orígenes de la flora fanerogámica de México. Acta Botanica Mexicana 14:3-21.

Rzedowski J. 1991b. El endemismo de la flora fanerogámica mexicana: una apreciación analítica preliminar. Acta Botanica Mexicana 15:47-64.

Rzedowski J. 1997. Los principales colectores botánicos de Guanajuato, Querétaro y norte de Michoacán. Fascículo complementario XVII. Flora del Bajío y de Regiones Adyacentes. Instituto de Ecología A. C. Centro regional del Bajío, Pátzcuaro.

Rzedowski J. 2001. Principales comunidades vegetales. En: Calderón de Rzedowski G. y Rzedowski J. Eds. Flora Fanerogámica del Valle de México, pp. 32-38, Instituto de Ecología y Comisión Nacional para el Conocimiento y Uso de la Biodiversidad, Pátzcuaro.

Rzedowski J. y Calderón de Rzedowski G. 1989. Sinopsis numérica de la flora fanerogámica del Valle de México. Acta Botanica Mexicana 8: 15-30.

Rzedowski J. y Calderón de Rzedowski G. (Eds.).1991. Presentación Guía para los autores y normas editoriales. Fascículo 1. Flora del Bajío y de Regiones Adyacentes. Instituto de Ecología A. C. Centro Regional del Bajío, Pátzcuaro.

Rzedowski J., Vibrans H. y Calderón de Rzedowski G. 2003. Senecio inaequidens DC. (Compositae, Senecioneae), una maleza perjudicial introducida en México. Acta Botanica Mexicana 63:83-96. 
Quero H.J. 1984. La vegetación de las serranías de la cuenca alta del Río de la Laja, Guanajuato. Anales del Instituto de Biología serie Botánica 47:73-99.

Sánchez S., Flores A., Cruz-Leyva I.A. y Velázquez A. 2009. Estado y transformación de los ecosistemas terrestres por causas humanas. En: Capital Natural de México, vol. II: Estado de Conservación y Tendencias de Cambio, pp. 75-129, Comisión Nacional para el Conocimiento y Uso de la Biodiversidad, México, D.F.

Scheinvar L. 2005. Flora Cactológica del Estado de Querétaro: Riqueza y Diversidad. Fondo de Cultura Económica, México, D.F.

SEMARNAT [Secretaría del Medio Ambiente y Recursos Naturales]. 2010. Norma Oficial Mexicana NOM-059-SEMARNAT-2010, Protección ambiental - Especies nativas de México de flora y fauna silvestres - Categorías de riesgo y especificaciones para su inclusión, exclusión o cambio - Lista de especies en riesgo. Diario Oficial de la Federación. 2da Sección, 30 de diciembre de 2010.

Serrano V., Sánchez F. y Pelz R. 1992. Plantas medicinales de Amealco, Qro.: recetario. Universidad Autónoma de Querétaro, Querétaro.

Styles B. 1993. Genus Pinus: A Mexican preview. En: Ramamoorthy, T. P., Bye R., Lot A. y Fa J. Eds. Biological Diversity of Mexico: Origins and Distribution, pp. 397-420, Oxford University Press, Nueva York.

Suárez-Ramos G., Serrano-Cárdenas V., Balderas-Aguilar P. y Pelz-Marín R. 2004. Atlas de Malezas Arvenses del Estado de Querétaro, Universidad Autónoma de Querétaro y Comisión Nacional para el Conocimiento y Uso de la Biodiversidad, Querétaro.

Toledo V.M. y Ordoñez M.J. 1993. The biodiversity scenario of Mexico: a review of terrestrial habitats. En: Ramamoorthy, T.
P., Bye R., Lot A. y Fa J. Eds. Biological Diversity of Mexico: Origins and Distribution, pp. 757-777, Oxford University Press, Nueva York.

Valencia A.S. 2004. Diversidad del género Quercus (Fagaceae) en México. Boletín de la Sociedad Botánica de México 75:33-53.

Velázquez A., Mas J.F., Díaz-Gallegos J.R., Mayorga-Saucedo R. y Alcántara P.C. 2002. Patrones y tasas de cambio del uso del suelo en México. Gaceta Ecológica 62:21-37.

Villarreal J.A. 1998. Gentianaceae. Fascículo 65. Flora del Bajío y de Regiones Adyacentes. Instituto de Ecología A. C., Centro Regional del Bajío, Pátzcuaro.

Villaseñor J.L. 2003. Diversidad y distribución de las magnoliphyta de México. Interciencia 28:160-167.

Villaseñor J.L. 2004. Los géneros de plantas vasculares de la flora de México. Boletín de la Sociedad Botánica de México 75:105135.

Villaseñor J.L. y Espinosa-García F.J. 1998. Catálogo de Malezas de México. Universidad Nacional Autónoma de México y Fondo de Cultura Económica, México, D.F.

Villaseñor J.L. y Espinosa-García F.J. 2004. The alien flowering plants of Mexico. Diversity and Distribution 10:113-123.

Villaseñor J.L. y Ortiz E. 2014. Biodiversidad de las plantas con flores (División Magnoliophyta) en México. Revista Mexicana de Biodiversidad 85:134-142.

Villaseñor J.L., Ortiz E. y Redonda-Martínez R. 2008. Catálogo de Autores de Plantas Vasculares de México. Instituto de BiologíaUniversidad Nacional Autónoma de México-Comisión para el Conocimiento y Uso de la Biodiversidad, México, D.F.

Zamudio S., Rzedowski J., Carranza E. y Calderón de Rzedowski G. 1992. La Vegetación del Estado de Querétaro. Consejo de Ciencia y Tecnología del Estado de Querétaro e Instituto de Ecología, Centro Regional del Bajío, Querétaro.

Recibido: 18 de junio de 2014

Aceptado: 1 de septiembre de 2014 
Apéndice. Catálogo de las plantas vasculares de la Sierra el Rincón, Querétaro y Michoacán, México. FORMAS DE VIDA Cf = caméfita, Ep = epífita, Fc = fanerófita cespitosa, $\mathrm{Fe}=$ fanerófita escaposa, Gf = geófita, $\mathrm{Hc}=$ hemicriptófita, $\mathrm{Hi}=$ hidrófita, $\mathrm{Li}=$ liana, $\mathrm{Pa}=$ parásita, $\mathrm{Tf}=$ terófita; $\mathrm{HÁBITO} \mathrm{a}=$ arbusto, $\mathrm{Ar}=$ árbol, $\mathrm{H}=$ Hierba; $\mathrm{N}=$ nativa, $\mathrm{I}=$ introducida; $\mathrm{X}=$ maleza o especie indicadora de disturbio; DISTRIBUCIÓN AM = amplia distribución en América, AN = México y las Antillas, CO = amplia distribución mundial, e = endémica a México, MC = de México a Centroamérica, MS = de México a Sudamérica, Na = de México a Norteamérica; ENDEMISMO cM = endémica al centro de México, $M=$ de amplia distribución en México; ESTATUS DE CONSERVACIÓN DE ACUERDO A LA NOM-059-SEMARNAT-2010 A = amenazada, Pr = protección especial.

\begin{tabular}{|c|c|c|c|c|c|c|c|}
\hline Grupo/Familia/Especie & $\begin{array}{c}\text { Forma } \\
\text { de vida }\end{array}$ & Hábito & $\begin{array}{c}\text { Nativa/ } \\
\text { Introducida }\end{array}$ & $\begin{array}{c}\text { Maleza o } \\
\text { Indicadora } \\
\text { de disturbio }\end{array}$ & $\begin{array}{l}\text { Distri- } \\
\text { bución }\end{array}$ & $\begin{array}{l}\text { Ende- } \\
\text { mismo }\end{array}$ & $\begin{array}{c}\text { NOM-059- } \\
\text { SEMARNAT- } \\
2010\end{array}$ \\
\hline
\end{tabular}

\section{PTERIDOPHYTA}

\section{Aspleniaceae}

Asplenium monanthes L.

Athyriaceae

Cystopteris fragilis (L.) Bernh.

Woodsia mollis (Kaulf.) J. Sm.

Blechnaceae

Woodwardia spinulosa M. Martens \& Galeotti

Dennstaedtiaceae

Pteridium aquilinum var. feei (W. Schaffn.

ex Fée) Maxon

\section{Dryopteridaceae}

Dryopteris cinnamomea (Cav.) C. Chr.

Elaphoglossum petiolatum (Sw.) Urb.

Equisetaceae

Equisetum hyemale subsp. affine (Engelm.)

Calder \& Roy L. Taylor

\section{Polypodiaceae}

Phlebodium pseudoaureum (Cav.) Lellinger

Pleopeltis polylepis (Roem. ex Kunze)

T. Moore var. polylepis

Polypodium madrense J. Sm.

Polypodium plebeium Schltdl. \& Cham.

Polypodium thyssanolepis A. Braun ex Klotzsch

Pteridaceae

Adiantum andicola Liebm.

Adiantum capillus-veneris L.

Adiantum poiretii Wikstr.

Astrolepis sinuata (Lag. ex Sw.) D.M. Benham

\& Windham

Cheilanthes bonariensis (Willd.) Proctor

Cheilanthes myriophylla Desv.

Gaga kaulfussii (Kunze) Fay W. Li \& Windham

Gaga marginata (Kunth) Fay W. Li \& Windham

Pellaea cordifolia (Sessé \& Moc.) A.R. Sm.

Pellaea ovata (Desv.) Weath.

Pellaea ternifolia (Cav.) Link subsp. ternifolia

Selaginellaceae

Selaginella pallescens (C. Presl) Spring

\begin{tabular}{|c|c|c|c|}
\hline $\mathrm{Hc}$ & $\mathrm{H}$ & $\mathrm{N}$ & - \\
\hline $\mathrm{Hc}$ & $\mathrm{H}$ & $\mathrm{N}$ & - \\
\hline $\mathrm{Hc}$ & $\mathrm{H}$ & $\mathrm{N}$ & - \\
\hline FC & $\mathrm{a}$ & $\mathrm{N}$ & - \\
\hline $\mathrm{Hc}$ & $\mathrm{H}$ & $\mathrm{N}$ & $x$ \\
\hline $\mathrm{Hc}$ & $\mathrm{H}$ & $\mathrm{N}$ & - \\
\hline EP & $\mathrm{H}$ & $\mathrm{N}$ & - \\
\hline $\mathrm{Hi}$ & $\mathrm{H}$ & $\mathrm{N}$ & - \\
\hline $\mathrm{Hc}$ & $\mathrm{H}$ & $\mathrm{N}$ & - \\
\hline $\mathrm{Hc}$ & $\mathrm{H}$ & $\mathrm{N}$ & - \\
\hline EP & $\mathrm{H}$ & $\mathrm{N}$ & - \\
\hline $\mathrm{Hc}$ & $\mathrm{H}$ & $\mathrm{N}$ & - \\
\hline $\mathrm{Hc}$ & $\mathrm{H}$ & $\mathrm{N}$ & - \\
\hline $\mathrm{Hc}$ & $\mathrm{H}$ & $\mathrm{N}$ & - \\
\hline $\mathrm{Hc}$ & $\mathrm{H}$ & $\mathrm{N}$ & - \\
\hline $\mathrm{Hc}$ & $\mathrm{H}$ & $\mathrm{N}$ & - \\
\hline $\mathrm{Hc}$ & $\mathrm{H}$ & $\mathrm{N}$ & - \\
\hline $\mathrm{Hc}$ & $\mathrm{H}$ & $\mathrm{N}$ & - \\
\hline $\mathrm{Hc}$ & $\mathrm{H}$ & $\mathrm{N}$ & - \\
\hline $\mathrm{Hc}$ & $\mathrm{H}$ & $\mathrm{N}$ & - \\
\hline $\mathrm{Hc}$ & $\mathrm{H}$ & $\mathrm{N}$ & - \\
\hline $\mathrm{Hc}$ & $\mathrm{H}$ & $\mathrm{N}$ & - \\
\hline $\mathrm{Hc}$ & $\mathrm{H}$ & $\mathrm{N}$ & - \\
\hline $\mathrm{Hc}$ & $\mathrm{H}$ & $\mathrm{N}$ & - \\
\hline $\mathrm{Hc}$ & $\mathrm{H}$ & $\mathrm{N}$ & - \\
\hline
\end{tabular}

\section{PINOPHYTA}

Cupressaceae

Cupressus lusitanica Mill.

Pinaceae

Pinus hartwegii Lindl.

Fe

$\mathrm{Fe}$
Ar

Ar
N

N
$\mathrm{CO}$

$\mathrm{CO}$

MC

$M C$

$\mathrm{Na}$

MS

$\mathrm{CO}$

MS

e

e

MS

MS

MS

$\mathrm{CO}$

$\mathrm{CO}$

MS

MS

MS

MS

MS

$\mathrm{Na}$

MS

MS

MS 
Apéndice. Continuación.

Grupo/Familia/Especie

\section{Forma}

Hábito

\section{Nativa/ Introducida}

Distri-

Maleza o de disturbio
Ende-

bución mismo SEMARNAT-

NOM-0592010

Pinus leiophylla Schiede ex Schltdl. \& Cham.

Pinus montezumae Lamb.

Pinus patula Schltdl. \& Cham.

Pinus pseudostrobus Lindl.

Pinus teocote Schltdl. \& Cham.

$\mathrm{Fe}$

$\mathrm{Fe}$

$\mathrm{Fe}$

$\mathrm{Fe}$

$\mathrm{Fe}$

$\mathrm{Ar}$
$\mathrm{Ar}$
$\mathrm{Ar}$
$\mathrm{Ar}$
$\mathrm{Ar}$

$\mathrm{HC}$

$\mathrm{H}$

N

e

M

\section{Eudicotyledoneae}

Acanthaceae

Dyschoriste microphylla Kuntze

Apiaceae

Arracacia aegopodioides (Kunth) J.M. Coult. \& Rose

Arracacia macvaughii Mathias \& Constance

Cyclospermum leptophyllum (Pers.) Sprague ex Britton \& P. Wilson

Donnellsmithia mexicana (B.L. Rob.) Mathias

\& Constance.

Eryngium columnare Hemsl.

Eryngium heterophyllum Engelm.

Eryngium serratum Cav.

Apocynaceae

Asclepias fournieri Woodson

Asclepias linaria Cav.

Asclepias pringlei (Greenm.) Woodson

Asteraceae

Acmella repens (Walter) Rich.

Acourtia hidalgoana B.L. Turner

Acourtia turbinata (Lex.) DC.

Ageratina choricephala (B.L. Rob.) R.M. King \& H. Rob.

Ageratina glabrata (Kunth) R.M. King \& H. Rob. Ageratina havanensis (Kunth) R.M. King \& H. Rob.

Ageratum corymbosum Zuccagni

Baccharis conferta Kunth

Baccharis heterophylla Kunth

Baccharis pteronioides DC.

Bidens bigelovii var. angustiloba (DC.) Ballard ex Melchert

Bidens schaffneri (A. Gray) Sherff

Brickellia nutanticeps S.F. Blake

Brickellia pedunculosa (DC.) Harc. \& Beaman

\begin{tabular}{|c|c|}
\hline $\mathrm{Hc}$ & $\mathrm{H}$ \\
\hline $\mathrm{Hc}$ & $\mathrm{H}$ \\
\hline $\mathrm{Hc}$ & $\mathrm{H}$ \\
\hline $\mathrm{Tf}$ & $\mathrm{H}$ \\
\hline $\mathrm{Tf}$ & $\mathrm{H}$ \\
\hline $\mathrm{Hc}$ & $\mathrm{H}$ \\
\hline $\mathrm{Hc}$ & $\mathrm{H}$ \\
\hline $\mathrm{Hc}$ & $\mathrm{H}$ \\
\hline $\mathrm{Hc}$ & $\mathrm{H}$ \\
\hline $\mathrm{Cf}$ & $\mathrm{H}$ \\
\hline $\mathrm{Hc}$ & $\mathrm{H}$ \\
\hline $\mathrm{Hi}$ & $\mathrm{H}$ \\
\hline $\mathrm{Hc}$ & $\mathrm{H}$ \\
\hline $\mathrm{Hc}$ & $\mathrm{H}$ \\
\hline $\mathrm{Cf}$ & $\mathrm{H}$ \\
\hline $\mathrm{Fc}$ & $\mathrm{a}$ \\
\hline $\mathrm{Fc}$ & $\mathrm{a}$ \\
\hline $\mathrm{Hc}$ & $\mathrm{H}$ \\
\hline $\mathrm{Fc}$ & $\mathrm{a}$ \\
\hline $\mathrm{Fc}$ & $\mathrm{a}$ \\
\hline FC & $\mathrm{a}$ \\
\hline $\mathrm{Tf}$ & $\mathrm{H}$ \\
\hline $\mathrm{Hi}$ & $\mathrm{H}$ \\
\hline $\mathrm{Cf}$ & $\mathrm{H}$ \\
\hline $\mathrm{Cf}$ & $\mathrm{H}$ \\
\hline $\mathrm{Hc}$ & $\mathrm{H}$ \\
\hline $\mathrm{Hc}$ & $\mathrm{H}$ \\
\hline $\mathrm{Hc}$ & $\mathrm{H}$ \\
\hline $\mathrm{Tf}$ & $\mathrm{H}$ \\
\hline
\end{tabular}

Carphochaete grahamii A. Gray

Chaptalia lyrata (Willd.) Spreng.

Cirsium pinetorum Greenm.

Conyza canadensis (L.) Cronquist

Conyza microcephala Hemsl.

$N$
$N$
$N$
$N$

$\begin{array}{cc}\mathrm{Na} & - \\ \mathrm{MC} & - \\ \mathrm{e} & \mathrm{M} \\ \mathrm{MC} & - \\ \mathrm{e} & \mathrm{M}\end{array}$

-


Apéndice. Continuación.

\begin{tabular}{|c|c|c|c|c|c|c|c|}
\hline Grupo/Familia/Especie & $\begin{array}{l}\text { Forma } \\
\text { de vida }\end{array}$ & Hábito & $\begin{array}{l}\text { Nativa/ } \\
\text { Introducida }\end{array}$ & $\begin{array}{c}\text { Maleza o } \\
\text { Indicadora } \\
\text { de disturbio }\end{array}$ & $\begin{array}{l}\text { Distri- } \\
\text { bución }\end{array}$ & $\begin{array}{l}\text { Ende- } \\
\text { mismo }\end{array}$ & $\begin{array}{c}\text { NOM-059- } \\
\text { SEMARNAT- } \\
2010\end{array}$ \\
\hline Cosmos bipinnatus Cav. & Tf & $\mathrm{H}$ & $\mathrm{N}$ & $\mathrm{x}$ & $\mathrm{Am}$ & - & - \\
\hline Cosmos parviflorus (Jacq.) Pers. & Tf & $\mathrm{H}$ & $\mathrm{N}$ & $\mathrm{x}$ & $\mathrm{Na}$ & - & - \\
\hline Dahlia coccinea Cav. & $\mathrm{Hc}$ & $\mathrm{H}$ & $\mathrm{N}$ & $\mathrm{x}$ & MC & - & - \\
\hline Dyssodia papposa (Vent.) Hitchc. & Tf & $\mathrm{H}$ & $\mathrm{N}$ & $x$ & Am & - & - \\
\hline Erigeron janivultus G.L. Nesom & $\mathrm{Hc}$ & $\mathrm{H}$ & $\mathrm{N}$ & - & $\mathrm{e}$ & M & - \\
\hline Galinsoga parviflora Cav. & Tf & $\mathrm{H}$ & $\mathrm{N}$ & $x$ & $\mathrm{CO}$ & - & - \\
\hline Galinsoga quadriradiata Ruiz \& Pav. & Tf & $\mathrm{H}$ & $\mathrm{N}$ & $\mathrm{x}$ & $\mathrm{Am}$ & - & - \\
\hline Gamochaeta americana (Mill.) Wedd. & Tf & $\mathrm{H}$ & $\mathrm{N}$ & $x$ & $\mathrm{Am}$ & - & - \\
\hline Grindelia inuloides Willd. & $\mathrm{Hc}$ & $\mathrm{H}$ & $\mathrm{N}$ & $x$ & $\mathrm{e}$ & $\mathrm{cM}$ & - \\
\hline Hieracium dysonymum S.F. Blake & $\mathrm{Hc}$ & $\mathrm{H}$ & $\mathrm{N}$ & - & e & M & - \\
\hline Hieracium pringlei A. Gray & $\mathrm{Hc}$ & $\mathrm{H}$ & $\mathrm{N}$ & - & AM & - & - \\
\hline lostephane heterophylla (Cav.) Benth. & $\mathrm{Hc}$ & $\mathrm{H}$ & $\mathrm{N}$ & - & $\mathrm{e}$ & M & - \\
\hline Laennecia confusa (Cronquist) G.L. Nesom & $\mathrm{Hc}$ & $\mathrm{H}$ & $\mathrm{N}$ & - & MC & - & - \\
\hline Montanoa grandiflora DC. & Fc & a & $\mathrm{N}$ & $\mathrm{x}$ & e & $\mathrm{cM}$ & - \\
\hline Packera sanguisorbae (DC.) C. Jeffrey & $\mathrm{Hc}$ & $\mathrm{H}$ & $\mathrm{N}$ & - & e & M & - \\
\hline Perymenium buphthalmoides DC. & $\mathrm{Hc}$ & $\mathrm{H}$ & $\mathrm{N}$ & - & $\mathrm{e}$ & M & - \\
\hline Piqueria trinervia Cav. & $\mathrm{Hc}$ & $\mathrm{H}$ & $\mathrm{N}$ & $\mathrm{X}$ & An & - & - \\
\hline Psacalium sinuatum (Cerv.) H.Rob. \& Brettell & $\mathrm{Hc}$ & $\mathrm{H}$ & $\mathrm{N}$ & - & $\mathrm{e}$ & M & - \\
\hline Pseudognaphalium canescens (DC.) Anderb. & $\mathrm{Hc}$ & $\mathrm{H}$ & $\mathrm{N}$ & - & $\mathrm{Na}$ & - & - \\
\hline Pseudognaphalium chartaceum (Greenm.) Anderb. & Tf & $\mathrm{H}$ & $\mathrm{N}$ & $\mathrm{x}$ & $\mathrm{e}$ & M & - \\
\hline $\begin{array}{l}\text { Pseudognaphalium luteoalbum (L.) Hilliard } \\
\text { \& B.L. Burtt }\end{array}$ & Tf & $\mathrm{H}$ & I & $\mathrm{x}$ & $\mathrm{CO}$ & - & - \\
\hline Pseudognaphalium oxyphyllum (DC.) Kirp. & Tf & $\mathrm{H}$ & $\mathrm{N}$ & $x$ & AM & - & - \\
\hline Pseudognaphalium purpurascens (DC.) Anderb. & Tf & $\mathrm{H}$ & $\mathrm{N}$ & - & e & M & - \\
\hline Roldana barba-johannis (DC.) H. Rob. \& Brettell & Fc & a & $\mathrm{N}$ & $\mathrm{X}$ & MC & - & - \\
\hline Roldana lineolata (DC.) H. Rob. \& Brettell & $\mathrm{Cf}$ & $\mathrm{H}$ & $\mathrm{N}$ & - & $\mathrm{e}$ & M & - \\
\hline Roldana michoacana (B.L. Rob.) H. Rob. \& Brettell & $\mathrm{Hc}$ & $\mathrm{H}$ & $\mathrm{N}$ & - & e & $\mathrm{cM}$ & - \\
\hline Roldana sessilifolia (Hook. \& Arn.) H. Rob. \& Brettell & $\mathrm{Hc}$ & $\mathrm{H}$ & $\mathrm{N}$ & - & e & M & - \\
\hline Schkuhria pinnata var. wislizeni (A. Gray) B.L. Turner & Tf & $\mathrm{H}$ & $\mathrm{N}$ & $\mathrm{x}$ & AM & - & - \\
\hline Senecio inaequidens DC. & Tf & $\mathrm{H}$ & I & $x$ & $\mathrm{CO}$ & - & - \\
\hline Stevia caracasana DC. & $\mathrm{Cf}$ & $\mathrm{H}$ & $\mathrm{N}$ & - & MS & - & - \\
\hline Stevia elatior Kunth & $\mathrm{Cf}$ & $\mathrm{H}$ & $\mathrm{N}$ & $x$ & MS & - & - \\
\hline Stevia eupatoria (Spreng.) Willd. & $\mathrm{Cf}$ & $\mathrm{H}$ & $\mathrm{N}$ & - & e & M & - \\
\hline Stevia incognita Grashoff & $\mathrm{Cf}$ & $\mathrm{H}$ & $\mathrm{N}$ & - & MS & - & - \\
\hline Stevia lucida Lag. var. lucida & FC & $\mathrm{a}$ & $\mathrm{N}$ & - & MS & - & - \\
\hline Stevia monardifolia Kunth & $\mathrm{Cf}$ & $\mathrm{H}$ & $\mathrm{N}$ & - & $\mathrm{e}$ & M & - \\
\hline Stevia origanoides Kunth & $\mathrm{Cf}$ & $\mathrm{H}$ & $\mathrm{N}$ & - & $\mathrm{e}$ & M & - \\
\hline Stevia pilosa Lag. & $\mathrm{Cf}$ & $\mathrm{H}$ & $\mathrm{N}$ & - & e & M & - \\
\hline Stevia serrata Cav. var. serrata & $\mathrm{Cf}$ & $\mathrm{H}$ & $\mathrm{N}$ & $x$ & $\mathrm{Am}$ & - & - \\
\hline Steviopsis adenosperma (Sch. Bip.) B.L. Turner & $\mathrm{Cf}$ & $\mathrm{H}$ & $\mathrm{N}$ & - & e & M & - \\
\hline Symphyotrichum subulatum (Michx.) G.L. Nesom & Tf & $\mathrm{H}$ & $\mathrm{N}$ & $\mathrm{x}$ & $\mathrm{Am}$ & - & - \\
\hline Tagetes erecta L. & Tf & $\mathrm{H}$ & $\mathrm{N}$ & $x$ & $\mathrm{Am}$ & - & - \\
\hline Tagetes foetidissima DC. & Tf & $\mathrm{H}$ & $\mathrm{N}$ & $\mathrm{x}$ & $\mathrm{MC}$ & - & - \\
\hline Tagetes lucida Cav. & $\mathrm{Hc}$ & $\mathrm{H}$ & $\mathrm{N}$ & $x$ & MC & - & - \\
\hline Tagetes lunulata Ortega & Tf & $\mathrm{H}$ & $\mathrm{N}$ & $x$ & MS & - & - \\
\hline Tagetes micrantha Cav. & Tf & $\mathrm{H}$ & $\mathrm{N}$ & $x$ & $\mathrm{Na}$ & - & - \\
\hline Taraxacum officinale F.H. Wigg. & $\mathrm{Hc}$ & $\mathrm{H}$ & I & $x$ & $\mathrm{CO}$ & - & - \\
\hline Viguiera sessilifolia DC. & $\mathrm{Hc}$ & $\mathrm{H}$ & $\mathrm{N}$ & - & e & $\mathrm{cM}$ & - \\
\hline $\begin{array}{l}\text { Viguiera urticiformis (DC.) Hemsl. } \\
\text { Begoniaceae }\end{array}$ & $\mathrm{Hc}$ & $\mathrm{H}$ & $\mathrm{N}$ & $x$ & e & $\mathrm{cM}$ & - \\
\hline Begonia gracilis Kunth & $\mathrm{Hc}$ & $\mathrm{H}$ & $\mathrm{N}$ & - & MC & - & - \\
\hline
\end{tabular}


Apéndice. Continuación.

Grupo/Familia/Especie
Forma de vida

Hábito

Nativa/
Introducida

Maleza o

Indicadora de disturbio
Distri- Ende-

bución mismo

mismo SEMARNAT2010

\section{Berberidaceae}

Berberis moranensis Schult. \& Schult. f.

Betulaceae

Alnus jorullensis Kunth subsp. jorullensis

Boraginaceae

Lithospermum calycosum (J.F. Macbr.) I.M. Johnst. Macromeria longiflora Sessé \& Moc. ex D. Don

Brassicaceae

Lepidium virginicum $\mathrm{L}$.

Raphanus raphanistrum $\mathrm{L}$.

Rorippa mexicana (DC.) Standl. \& Steyerm.

Cactaceae

Mammillaria rhodantha subsp. pringlei (J.M. Coult.)

D.R. Hunt

Opuntia robusta J.C. Wendl.

Opuntia tomentosa Salm-Dyck

\section{Campanulaceae}

Diastatea tenera (A. Gray) McVaugh

Lobelia gruina Cav.

Lobelia laxiflora Kunth

Lobelia nana Kunth

\section{Caryophyllaceae}

Arenaria bourgaei Hemsl.

Arenaria lanuginosa (Michx.) Rohrb. var. lanuginosa

Cornus excelsa Kunth

\section{Crassulaceae}

Altamiranoa mexicana (Schltdl.) Rose

Echeveria mucronata Schltdl.

Echeveria secunda Booth ex Lindl.

Sedum greggii Hemsl.

Cucurbitaceae

Sicyos deppei G. Don

Ericaceae

Arbutus tessellata P.D. Sorensen

Arbutus xalapensis Kunth

Arctostaphylos pungens Kunth
FC

$\mathrm{Fe}$

Hc H

Cf $\mathrm{H}$

Tf

Tf

$\mathrm{Hi}$

Cf

Cf $\quad \mathrm{H}$

Fc a

FC $\quad a$

Tf

$\mathrm{Hc}$

$\mathrm{Hi}$

$\mathrm{Hc}$

$\mathrm{HC} \quad \mathrm{H}$

$\mathrm{Hc} \quad \mathrm{H}$

Tf $\quad H$

Tf $\quad H$

$\mathrm{HC} \quad \mathrm{H}$

$\mathrm{HC} \quad \mathrm{H}$

Tf H

Cf $\quad \mathrm{H}$

$\mathrm{Fe} \quad \mathrm{Ar}$

$\mathrm{Hc}$

$\mathrm{Hc}$

$\mathrm{Li}$

FC

a

Ar N

N

$\mathrm{N}$

H

$\mathrm{H}$

$\mathrm{H}$

H N

N

$\mathrm{H}$

$\mathrm{H}$

H N

$\mathrm{N}$

N

$N$

$\mathrm{N}$

$N$

I

H N

Ar N

$\mathrm{H} \quad \mathrm{N}$

$\begin{array}{lll} & & \\ \mathrm{HC} & \mathrm{H} & \mathrm{N} \\ \mathrm{HC} & \mathrm{H} & \mathrm{N} \\ \mathrm{HC} & \mathrm{H} & \mathrm{N} \\ \mathrm{HC} & \mathrm{H} & \mathrm{N}\end{array}$

Li $\mathrm{H} \quad \mathrm{N}$

Fe $\quad A r$

Fe $\mathrm{Ar}$

Fc a
N

M

e M

MC

AM

e

$\mathrm{CO}$

$\mathrm{CO}$

$\mathrm{MC}$

e

$\mathrm{cM}$

$\operatorname{Pr}$

$\mathrm{Na}$

AM

MC

$\mathrm{Na}$

Am

Am

MS

Am

Am

MC

e

$\mathrm{Na}$

$\mathrm{CO}$

AM

e

$\mathrm{CO}$

e

e

$M$

$M$

MC

e $\mathrm{cM}$

e

e

e

$M$

$\mathrm{CM}$

$\mathrm{cM}$

e $M$

N

$N$

$N$

M -

$$
\begin{aligned}
& - \\
& - \\
& - \\
& - \\
& -
\end{aligned}
$$$$
-
$$$$
\text { - }
$$$$
-
$$$$
-
$$$$
-
$$$$
\text { - }
$$$$
\text { - }
$$$$
-
$$$$
\text { - }
$$$$
-
$$

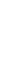$$
\text { r }
$$ 
Apéndice. Continuación.

\begin{tabular}{|c|c|c|c|c|c|c|c|}
\hline Grupo/Familia/Especie & $\begin{array}{l}\text { Forma } \\
\text { de vida }\end{array}$ & Hábito & $\begin{array}{l}\text { Nativa/ } \\
\text { Introducida }\end{array}$ & $\begin{array}{c}\text { Maleza o } \\
\text { Indicadora } \\
\text { de disturbio }\end{array}$ & $\begin{array}{l}\text { Distri- } \\
\text { bución }\end{array}$ & $\begin{array}{l}\text { Ende- } \\
\text { mismo }\end{array}$ & $\begin{array}{c}\text { NOM-059- } \\
\text { SEMARNAT- } \\
2010\end{array}$ \\
\hline Monotropa hypopitys L. & $\mathrm{Hc}$ & $\mathrm{H}$ & $\mathrm{N}$ & - & $\mathrm{CO}$ & - & - \\
\hline \multicolumn{8}{|l|}{ Euphorbiaceae } \\
\hline Euphorbia anychioides Boiss. & $\mathrm{Hc}$ & $\mathrm{H}$ & $\mathrm{N}$ & $X$ & MC & - & - \\
\hline Euphorbia furcillata Kunth var. furcillata & $\mathrm{Hc}$ & $\mathrm{H}$ & $\mathrm{N}$ & - & MC & - & - \\
\hline Euphorbia hirta L. & Tf & $\mathrm{H}$ & $\mathrm{N}$ & $\mathrm{X}$ & MC & - & - \\
\hline Tragia nepetifolia Cav. & $\mathrm{Hc}$ & $\mathrm{H}$ & $\mathrm{N}$ & - & AM & - & - \\
\hline \multicolumn{8}{|l|}{ Fabaceae } \\
\hline Calliandra grandiflora (L'Hér.) Benth. & FC & $\mathrm{a}$ & $\mathrm{N}$ & - & MC & - & - \\
\hline Cologania broussonetii (Balb.) DC. & $\mathrm{Li}$ & $\mathrm{H}$ & $\mathrm{N}$ & $x$ & MS & - & - \\
\hline Desmodium aparines (Link) DC. & $\mathrm{Li}$ & $\mathrm{H}$ & $\mathrm{N}$ & - & Am & - & - \\
\hline Desmodium grahamii A. Gray & $\mathrm{Li}$ & $\mathrm{H}$ & $\mathrm{N}$ & - & $\mathrm{Na}$ & - & - \\
\hline Desmodium pringlei S. Watson & $\mathrm{Hc}$ & $\mathrm{H}$ & $\mathrm{N}$ & - & $M C$ & - & - \\
\hline Desmodium uncinatum (Jacq.) DC. & $\mathrm{Li}$ & $\mathrm{H}$ & $\mathrm{N}$ & - & Am & - & - \\
\hline Lupinus stipulatus J.Agardh & $\mathrm{Hc}$ & $\mathrm{H}$ & $\mathrm{N}$ & - & $\mathrm{e}$ & $\mathrm{cM}$ & - \\
\hline Macroptilium gibbosifolium (Ortega) A. Delgado & $\mathrm{Hc}$ & $\mathrm{H}$ & $\mathrm{N}$ & $X$ & Am & - & - \\
\hline Mimosa biuncifera Benth. & Fc & $\mathrm{a}$ & $\mathrm{N}$ & $\mathrm{X}$ & $\mathrm{Na}$ & - & - \\
\hline Painteria leptophylla (DC.) Britton \& Rose & FC & $\mathrm{a}$ & $\mathrm{N}$ & $X$ & $\mathrm{e}$ & M & - \\
\hline Phaseolus pluriflorus Maréchal, Mascherpa \& Stainier & $\mathrm{Li}$ & $\mathrm{H}$ & $\mathrm{N}$ & $X$ & $\mathrm{e}$ & M & - \\
\hline Trifolium mexicanum Hemsl. & $\mathrm{Hc}$ & $\mathrm{H}$ & $\mathrm{N}$ & $X$ & $\mathrm{MC}$ & - & - \\
\hline Zornia thymifolia Kunth & $\mathrm{Hc}$ & $\mathrm{H}$ & $\mathrm{N}$ & - & MC & - & - \\
\hline \multicolumn{8}{|l|}{ Fagaceae } \\
\hline Quercus candicans Née & $\mathrm{Fe}$ & Ar & $\mathrm{N}$ & - & $\mathrm{MC}$ & - & - \\
\hline Quercus castanea Née & $\mathrm{Fe}$ & $\mathrm{Ar}$ & $\mathrm{N}$ & - & $\mathrm{e}$ & $M$ & - \\
\hline Quercus crassifolia Bonpl. & $\mathrm{Fe}$ & $\mathrm{Ar}$ & $\mathrm{N}$ & - & $\mathrm{MC}$ & - & - \\
\hline Quercus crassipes Bonpl. & $\mathrm{Fe}$ & Ar & $\mathrm{N}$ & - & $\mathrm{e}$ & $\mathrm{cM}$ & - \\
\hline Quercus eduardi Trel. & $\mathrm{Fe}$ & $\mathrm{Ar}$ & $\mathrm{N}$ & - & $\mathrm{e}$ & M & - \\
\hline Quercus laeta Liebm. & $\mathrm{Fe}$ & $\mathrm{Ar}$ & $\mathrm{N}$ & - & $\mathrm{e}$ & M & - \\
\hline Quercus laurina Bonpl. & $\mathrm{Fe}$ & $\operatorname{Ar}$ & $\mathrm{N}$ & - & $\mathrm{e}$ & cM & - \\
\hline Quercus obtusata Bonpl. & $\mathrm{Fe}$ & Ar & $\mathrm{N}$ & - & $\mathrm{e}$ & M & - \\
\hline Quercus rugosa Née & $\mathrm{Fe}$ & $\operatorname{Ar}$ & $\mathrm{N}$ & - & AM & - & - \\
\hline \multicolumn{8}{|l|}{ Gentianaceae } \\
\hline Gentiana spathacea Kunth & $\mathrm{Hc}$ & $\mathrm{H}$ & $\mathrm{N}$ & 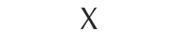 & $\mathrm{e}$ & M & $\operatorname{Pr}$ \\
\hline $\begin{array}{l}\text { Gentianopsis detonsa var. lanceolata (Benth.) } \\
\text { Villarreal et A.E. Estrada }\end{array}$ & Tf & $\mathrm{H}$ & $\mathrm{N}$ & - & $\mathrm{e}$ & M & - \\
\hline \multicolumn{8}{|l|}{ Geraniaceae } \\
\hline Erodium cicutarium (L.) L'Hér. ex Aiton & Tf & $\mathrm{H}$ & 1 & $x$ & $\mathrm{CO}$ & - & - \\
\hline Geranium bellum Rose & $\mathrm{Hc}$ & $\mathrm{H}$ & $\mathrm{N}$ & - & $\mathrm{e}$ & M & - \\
\hline Geranium seemannii Peyr. & $\mathrm{Hc}$ & $\mathrm{H}$ & $\mathrm{N}$ & $X$ & MS & - & - \\
\hline \multicolumn{8}{|l|}{ Hypericaceae } \\
\hline Hypericum galinum S.F. Blake & $\mathrm{Hc}$ & $\mathrm{H}$ & $\mathrm{N}$ & 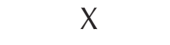 & $\mathrm{e}$ & $\mathrm{cM}$ & - \\
\hline Hypericum philonotis Schltdl. \& Cham. & Tf & $\mathrm{H}$ & $\mathrm{N}$ & - & MC & - & - \\
\hline Hypericum pauciflorum Kunth & $\mathrm{Hc}$ & $\mathrm{H}$ & $\mathrm{N}$ & - & $\mathrm{Na}$ & - & - \\
\hline \multicolumn{8}{|l|}{ Lamiaceae } \\
\hline Lepechinia caulescens (Ortega) Epling & $\mathrm{Hc}$ & $\mathrm{H}$ & $\mathrm{N}$ & - & $M C$ & - & - \\
\hline Prunella vulgaris $\mathrm{L}$. & $\mathrm{Hc}$ & $\mathrm{H}$ & $\mathrm{N}$ & - & $\mathrm{CO}$ & - & - \\
\hline Salvia elegans Vahl & Cf & $\mathrm{H}$ & $\mathrm{N}$ & - & $\mathrm{e}$ & M & - \\
\hline Salvia helianthemifolia Benth. & $\mathrm{Cf}$ & $\mathrm{H}$ & $\mathrm{N}$ & - & $\mathrm{e}$ & M & - \\
\hline Salvia laevis Benth. & Cf & $\mathrm{H}$ & $\mathrm{N}$ & - & $\mathrm{e}$ & M & - \\
\hline Salvia mexicana L. & Cf & $\mathrm{H}$ & $\mathrm{N}$ & $x$ & $\mathrm{e}$ & M & - \\
\hline Salvia patens Cav. & $\mathrm{Cf}$ & $\mathrm{H}$ & $\mathrm{N}$ & - & $\mathrm{e}$ & $\mathrm{cM}$ & - \\
\hline
\end{tabular}


Apéndice. Continuación.

\begin{tabular}{|c|c|c|c|c|c|c|c|}
\hline Grupo/Familia/Especie & $\begin{array}{l}\text { Forma } \\
\text { de vida }\end{array}$ & Hábito & $\begin{array}{l}\text { Nativa/ } \\
\text { Introducida }\end{array}$ & $\begin{array}{c}\text { Maleza o } \\
\text { Indicadora } \\
\text { de disturbio }\end{array}$ & $\begin{array}{l}\text { Distri- } \\
\text { bución }\end{array}$ & $\begin{array}{l}\text { Ende- } \\
\text { mismo }\end{array}$ & $\begin{array}{c}\text { NOM-059- } \\
\text { SEMARNAT- } \\
2010\end{array}$ \\
\hline Salvia reptans Jacq. & $\mathrm{Hc}$ & $\mathrm{H}$ & $\mathrm{N}$ & $x$ & $\mathrm{Na}$ & - & - \\
\hline Scutellaria coerulea Moc. \& Sessé ex Benth. & $\mathrm{Hc}$ & $\mathrm{H}$ & $\mathrm{N}$ & - & MC & - & - \\
\hline Stachys agraria Schltdl. \& Cham. & Tf & $\mathrm{H}$ & $\mathrm{N}$ & $x$ & AM & - & - \\
\hline Stachys coccinea Ortega & $\mathrm{Hc}$ & $\mathrm{H}$ & $\mathrm{N}$ & $x$ & AM & - & - \\
\hline \multicolumn{8}{|l|}{ Lentibulariaceae } \\
\hline Pinguicula moranensis var. neovolcanica Zamudio & $\mathrm{Hc}$ & $\mathrm{H}$ & $\mathrm{N}$ & - & $\mathrm{e}$ & $\mathrm{cM}$ & - \\
\hline \multicolumn{7}{|l|}{ Linaceae } & - \\
\hline $\begin{array}{l}\text { Linum mexicanum Kunth } \\
\text { Loranthaceae }\end{array}$ & $\mathrm{Hc}$ & $\mathrm{H}$ & $\mathrm{N}$ & - & $\mathrm{e}$ & $M$ & - \\
\hline $\begin{array}{l}\text { Cladocolea diversifolia (Benth.) Kuijt } \\
\text { Malpighiaceae }\end{array}$ & \multicolumn{6}{|c|}{ Malpighiaceae } & - \\
\hline $\begin{array}{l}\text { Gaudichaudia cynanchoides Kunth } \\
\text { Myrtaceae }\end{array}$ & \multicolumn{6}{|c|}{ Myrtaceae } & - \\
\hline $\begin{array}{l}\text { Eucalyptus camaldulensis Dehnh. } \\
\text { Onagraceae }\end{array}$ & $\mathrm{Fe}$ & $\mathrm{Ar}$ & I & $x$ & $\mathrm{CO}$ & - & - \\
\hline Fuchsia thymifolia Kunth & Fc & $\mathrm{a}$ & $\mathrm{N}$ & - & MC & - & - \\
\hline Lopezia racemosa Cav. & Tf & $\mathrm{H}$ & $\mathrm{N}$ & $X$ & MC & - & - \\
\hline Oenothera deserticola (Loes.) Munz & $\mathrm{Hc}$ & $\mathrm{H}$ & $\mathrm{N}$ & - & $\mathrm{e}$ & M & - \\
\hline Oenothera elata Kunth & $\mathrm{Hc}$ & $\mathrm{H}$ & $\mathrm{N}$ & $x$ & AM & - & - \\
\hline Oenothera pubescens Willd. ex Spreng. & $\mathrm{Hc}$ & $\mathrm{H}$ & $\mathrm{N}$ & $X$ & AM & - & - \\
\hline Oenothera rosea L'Hér. ex Aiton & Tf & $\mathrm{H}$ & $\mathrm{N}$ & $X$ & Am & - & - \\
\hline \multicolumn{8}{|l|}{ Orobanchaceae } \\
\hline Castilleja lithospermoides Kunth & $\mathrm{Cf}$ & $\mathrm{H}$ & $\mathrm{N}$ & - & $\mathrm{e}$ & M & - \\
\hline Castilleja nervata Eastw. & $\mathrm{Cf}$ & $\mathrm{H}$ & $\mathrm{N}$ & - & $\mathrm{e}$ & M & - \\
\hline Castilleja tenuiflora Benth. & $\mathrm{Cf}$ & $\mathrm{H}$ & $\mathrm{N}$ & $x$ & $\mathrm{e}$ & M & - \\
\hline Conopholis alpina Liebm. & $\mathrm{Pa}$ & $\mathrm{H}$ & $\mathrm{N}$ & - & AM & - & - \\
\hline Lamourouxia dasyantha (Cham. \& Schltdl.) W.R. Ernst & $\mathrm{Cf}$ & $\mathrm{H}$ & $\mathrm{N}$ & - & $\mathrm{e}$ & M & - \\
\hline Lamourouxia multifida Kunth & $\mathrm{Cf}$ & $\mathrm{H}$ & $\mathrm{N}$ & $x$ & MC & - & - \\
\hline $\begin{array}{l}\text { Lamourouxia rhinanthifolia Kunth } \\
\text { Oxalidaceae }\end{array}$ & \multicolumn{6}{|c|}{ Oxalidaceae } & - \\
\hline Oxalis corniculata L. & $\mathrm{Hc}$ & $\mathrm{H}$ & 1 & $x$ & $\mathrm{CO}$ & - & - \\
\hline Oxalis galeottii Turcz. & Gf & $\mathrm{H}$ & $\mathrm{N}$ & - & AM & - & - \\
\hline \multicolumn{8}{|l|}{ Passifloraceae } \\
\hline $\begin{array}{l}\text { Passiflora exsudans Zucc. } \\
\text { Phrymaceae }\end{array}$ & \multicolumn{6}{|c|}{ Phrymaceae } & - \\
\hline \multicolumn{7}{|l|}{ Phytolaccaceae } & - \\
\hline \multicolumn{7}{|l|}{ Plantaginaceae } & - \\
\hline Bacopa monnieri (L.) Wettst. & $\mathrm{Hc}$ & $\mathrm{H}$ & $\mathrm{N}$ & $x$ & $\mathrm{CO}$ & - & - \\
\hline Penstemon campanulatus (Cav.) Willd. & $\mathrm{Hc}$ & $\mathrm{H}$ & $\mathrm{N}$ & - & MC & - & - \\
\hline Penstemon miniatus subsp. apateticus (Straw) Straw & $\mathrm{Hc}$ & $\mathrm{H}$ & $\mathrm{N}$ & - & $\mathrm{e}$ & M & - \\
\hline Penstemon roseus (Cerv. ex Sweet) G. Don & $\mathrm{Hc}$ & $\mathrm{H}$ & $\mathrm{N}$ & $\mathrm{X}$ & $\mathrm{e}$ & M & - \\
\hline Plantago australis subsp. hirtella (Kunth) Rahn & $\mathrm{Hc}$ & $\mathrm{H}$ & $\mathrm{N}$ & $\mathrm{X}$ & Am & - & - \\
\hline Plantago nivea Kunth & $\mathrm{Hc}$ & $\mathrm{H}$ & $\mathrm{N}$ & $x$ & Am & - & - \\
\hline \multicolumn{8}{|l|}{ Polemoniaceae } \\
\hline Loeselia caerulea (Cav.) G. Don & $\mathrm{Hc}$ & $\mathrm{H}$ & $\mathrm{N}$ & $x$ & $\mathrm{e}$ & M & - \\
\hline Loeselia mexicana (Lam.) Brand & Cf & $\mathrm{H}$ & $\mathrm{N}$ & $\mathrm{X}$ & $\mathrm{e}$ & M & - \\
\hline
\end{tabular}


Apéndice. Continuación.

\begin{tabular}{|c|c|c|c|c|c|c|c|}
\hline Grupo/Familia/Especie & $\begin{array}{c}\text { Forma } \\
\text { de vida }\end{array}$ & Hábito & $\begin{array}{c}\text { Nativa/ } \\
\text { Introducida }\end{array}$ & $\begin{array}{c}\text { Maleza o } \\
\text { Indicadora } \\
\text { de disturbio }\end{array}$ & $\begin{array}{l}\text { Distri- } \\
\text { bución }\end{array}$ & $\begin{array}{l}\text { Ende- } \\
\text { mismo }\end{array}$ & $\begin{array}{l}\text { NOM-059- } \\
\text { SEMARNAT- } \\
2010\end{array}$ \\
\hline
\end{tabular}

\section{Polygalaceae}

Monnina ciliolata Sessé \& Moc. ex DC.

Polygonaceae

Polygonum hydropiperoides Michx.

Polygonum mexicanum Small

Ranunculaceae

Ranunculus hydrocharoides A. Gray

Ranunculus petiolaris var. arsenei (L.D. Benson)

T. Duncan

Thalictrum gibbosum Lecoy.

Rhamnaceae

Rhamnus microphylla Humb. \& Bonpl. ex Schult.

\section{Rosaceae}

Alchemilla aphanoides var. subalpestris (Rose)

L.M. Perry

Amelanchier denticulata (Kunth) K. Koch

Crataegus mexicana DC.

Prunus serotina subsp. capuli (Cav.) McVaugh

Rubiaceae

Bouvardia ternifolia (Cav.) Schltdl.

Crusea diversifolia (Kunth) W.R. Anderson

Crusea longiflora (Roem. \& Schult.) W.R.Anderson

Didymaea alsinoides (Schltdl. \& Cham) Standl.

Richardia tricocca subsp. tetracocca (M. Martens

\& Galeotti) W.H. Lewis \& R.L. Oliv.

\section{Santalaceae}

Arceuthobium vaginatum (Humb. \& Bonpl.

ex Willd.) J. Presl

Phoradendron velutinum (DC.) Oliv.

\section{Salicaceae}

Salix bonplandiana Kunth

Saxifragaceae

Heuchera orizabensis Hemsl.

Scrophulariaceae

Buddleja cordata Kunth

Limosella aquatica L.

Solanaceae

Bouchetia erecta DC. ex Dunal

Cestrum thyrsoideum Kunth

Jaltomata procumbens (Cav.) J.L. Gentry

Lycianthes moziniana (Dunal) Bitter

Physalis chenopodifolia Lam.

Physalis subrepens Waterf.

Solanum americanum Mill.

Solanum nigrescens M. Martens \& Galeotti

Tropaeolaceae

Tropaeolum majus L.

Verbenaceae

Glandularia teucriifolia (M. Martens \&

Galeotti) Umber

\begin{tabular}{|c|c|}
\hline Fc & $\mathrm{a}$ \\
\hline $\mathrm{Hi}$ & $\mathrm{H}$ \\
\hline $\mathrm{Hi}$ & $\mathrm{H}$ \\
\hline $\mathrm{Hi}$ & $\mathrm{H}$ \\
\hline $\mathrm{Hc}$ & $\mathrm{H}$ \\
\hline $\mathrm{Hc}$ & $\mathrm{H}$ \\
\hline $\mathrm{Fc}$ & $\mathrm{a}$ \\
\hline $\mathrm{Hc}$ & $\mathrm{H}$ \\
\hline Fc & $\mathrm{a}$ \\
\hline $\mathrm{Fe}$ & $\mathrm{Ar}$ \\
\hline $\mathrm{Fe}$ & $\mathrm{Ar}$ \\
\hline FC & $\mathrm{a}$ \\
\hline $\mathrm{Tf}$ & $\mathrm{H}$ \\
\hline $\mathrm{Tf}$ & $\mathrm{H}$ \\
\hline $\mathrm{Hc}$ & $\mathrm{H}$ \\
\hline $\mathrm{Hc}$ & $\mathrm{H}$ \\
\hline
\end{tabular}

N

$N$

N

$N$

N

N

N

$N$

$N$

$\mathrm{N}$

$N$

$N$

$N$

$N$

$\mathrm{HC} \quad \mathrm{H}$

$\mathrm{Pa}$

$\mathrm{Pa}$

Fe

HC

$\mathrm{Fe}$

$\mathrm{Hi}$

Hc H

Fc a

Hc H

$\mathrm{Hc} \quad \mathrm{H}$

$\mathrm{HC} \quad \mathrm{H}$

$\mathrm{HC} \quad \mathrm{H}$

Tf H

Tf H

Hc $\mathrm{H}$

Hc e

M

Am

AM

AM

$\mathrm{Na}$

e

cM

e

M

MS

X

X

MC

$\mathrm{MC}$

MC

$\mathrm{Na}$

AM

MS

e

e

CM

M

AM

MC

AM

e

cM

MC

$\mathrm{CO}$

Am

e

MC

e

e

e

$\mathrm{CO}$

Am

Am

X

X

$M C$ 
Apéndice. Continuación.

\begin{tabular}{|c|c|c|c|c|c|c|c|}
\hline Grupo/Familia/Especie & $\begin{array}{l}\text { Forma } \\
\text { de vida }\end{array}$ & Hábito & $\begin{array}{c}\text { Nativa/ } \\
\text { Introducida }\end{array}$ & $\begin{array}{c}\text { Maleza o } \\
\text { Indicadora } \\
\text { de disturbio }\end{array}$ & $\begin{array}{l}\text { Distri- } \\
\text { bución }\end{array}$ & $\begin{array}{l}\text { Ende- } \\
\text { mismo }\end{array}$ & $\begin{array}{c}\text { NOM-059- } \\
\text { SEMARNAT- } \\
2010\end{array}$ \\
\hline
\end{tabular}

Priva mexicana (L.) Pers.

Verbena carolina L.

Verbena menthifolia Benth.

Violaceae

Viola grahamii Benth.

\section{Monocotyledoneae}

Amaryllidaceae

Allium glandulosum Link \& Otto

Allium rhizomatum Wooton \& Standl.

Asparagaceae

Agave mapisaga Trel.

Dasylirion acrotrichum (Schiede) Zucc.

Echeandia durangensis (Greenm.) Cruden

(Baker) Cruden

Manfreda guttata (Jacobi \& C.D. Bouché) Rose

Milla biflora Cav.

Polianthes geminiflora (Lex.) Rose

\section{Bromeliaceae}

Tillandsia juncea (Ruiz \& Pav.) Poir.

Tillandsia recurvata (L.) L.

Tillandsia tortilis Klotzsch ex Baker

\section{Commelinaceae}

Commelina dianthifolia Delile

Tradescantia crassifolia Cav.

Tripogandra purpurascens (S. Schauer) Handlos

Cyperaceae

Bulbostylis juncoides (Vahl) Kük. ex Osten

Carex ciliaris Fernald

Carex psilocarpa Steud.

Cyperus manimae Kunth

Cyperus niger Ruiz \& Pav.

Cyperus pallidicolor (Kük.) G.C. Tucker

Cyperus seslerioides Kunth

Eleocharis ignota S. González \& Reznicek

Eleocharis montevidensis Kunth

Karinia mexicana (C.B. Clarke ex Britton)

Reznicek \& McVaugh

\section{Eriocaulaceae}

Eriocaulon benthamii Kunth

Hypoxidaceae

Hypoxis mexicana Schult. \& Schult. f.

Iridaceae

Nemastylis tenuis (Herb.) S. Watson

Tigridia multiflora (Baker) Ravenna

Juncaceae

Juncus acuminatus Michx.

Juncus bufonius L.

Juncus effusus L.

Juncus marginatus Rostk.

$\begin{array}{lllll}\text { Hc } & H & N & X & M C \\ H c & H & N & X & \text { AM } \\ \text { Hc } & H & N & X & \text { AM } \\ \text { Hc } & H & N & X & M C\end{array}$

$\begin{array}{llll}\text { Gf } & \mathrm{H} & \mathrm{N} & \mathrm{X} \\ \text { Gf } & \mathrm{H} & \mathrm{N} & - \\ \text { Fc } & \mathrm{a} & \mathrm{N} & - \\ \text { Fc } & \mathrm{a} & \mathrm{N} & - \\ \text { Hc } & \mathrm{H} & \mathrm{N} & - \\ \text { Gf } & \mathrm{H} & \mathrm{N} & - \\ \text { Gf } & \mathrm{H} & \mathrm{N} & - \\ \text { Gf } & \mathrm{H} & \mathrm{N} & \mathrm{X} \\ \text { Gf } & \mathrm{H} & \mathrm{N} & -\end{array}$

AM

$\mathrm{Na}$

e $M$

e $M$

e $\quad M$

e $\quad \mathrm{cM}$

e

AM

e

MS

Am

e

$\mathrm{cM}$

$\begin{array}{ll}\text { Ep } & \mathrm{H} \\ \mathrm{HC} & \mathrm{H}\end{array}$

Gf $\quad \mathrm{H}$

Gf $\mathrm{H}$

Tf H

$\mathrm{Na}$

AM

MS

$\mathrm{Hc} \quad \mathrm{H}$

$\mathrm{HC} \quad \mathrm{H}$

$\mathrm{Hc} \quad \mathrm{H}$

$\mathrm{Hc} \quad \mathrm{H}$

$\mathrm{Hi} \quad \mathrm{H}$

$\mathrm{HC} \quad \mathrm{H}$

$\mathrm{HC} \quad \mathrm{H}$

$\mathrm{Hi} \quad \mathrm{H}$

$\mathrm{Hi} \quad \mathrm{H}$

Hc H

Am

e

$M C$

AM

Am

$\mathrm{Am}$

Am

e

Am

e

$\mathrm{Hi}$

Gf

Gf

Gf

$\mathrm{H}$

$\mathrm{H}$

N

MC

$\mathrm{Na}$

e

e

$\mathrm{Hi} \quad \mathrm{H}$

$\mathrm{HI} \quad \mathrm{H}$

$\mathrm{Hi} \quad \mathrm{H}$

$\mathrm{Hi} H$
AM

$\mathrm{CO}$

$\mathrm{CO}$

Am 
Apéndice. Continuación.

\begin{tabular}{|c|c|c|c|c|c|c|c|}
\hline Grupo/Familia/Especie & $\begin{array}{l}\text { Forma } \\
\text { de vida }\end{array}$ & Hábito & $\begin{array}{l}\text { Nativa/ } \\
\text { Introducida }\end{array}$ & $\begin{array}{c}\text { Maleza o } \\
\text { Indicadora } \\
\text { de disturbio }\end{array}$ & $\begin{array}{l}\text { Distri- } \\
\text { bución }\end{array}$ & $\begin{array}{l}\text { Ende- } \\
\text { mismo }\end{array}$ & $\begin{array}{l}\text { NOM-059- } \\
\text { SEMARNAT- } \\
2010\end{array}$ \\
\hline Juncus microcephalus Kunth & $\mathrm{Hi}$ & $\mathrm{H}$ & $\mathrm{N}$ & - & MS & - & - \\
\hline Juncus tenuis Willd. & $\mathrm{Hi}$ & $\mathrm{H}$ & $\mathrm{N}$ & - & Am & - & - \\
\hline \multicolumn{8}{|l|}{ Orchidaceae } \\
\hline Bletia neglecta Sosa & Gf & $\mathrm{H}$ & $\mathrm{N}$ & - & $E$ & $\mathrm{cM}$ & - \\
\hline Corallorhiza maculata (Raf.) Raf. & Gf & $\mathrm{H}$ & $\mathrm{N}$ & - & AM & - & - \\
\hline Malaxis fastigiata (Rchb. f.) Kuntze & Gf & $\mathrm{H}$ & $\mathrm{N}$ & - & MC & - & - \\
\hline Malaxis soulei L.O. Williams & Gf & $\mathrm{H}$ & $\mathrm{N}$ & - & AM & - & - \\
\hline \multicolumn{8}{|l|}{ Poaceae } \\
\hline Agrostis avenacea J.F. Gmel. & $\mathrm{Hc}$ & $\mathrm{H}$ & 1 & $x$ & $\mathrm{CO}$ & - & - \\
\hline $\begin{array}{l}\text { Achnatherum constrictum (Hitchc.) Valdés-Reyna } \\
\text { \& Barkworth }\end{array}$ & $\mathrm{Hc}$ & $\mathrm{H}$ & $\mathrm{N}$ & - & $\mathrm{E}$ & M & - \\
\hline Aegopogon cenchroides Humb. \& Bonpl. ex Willd. & $\mathrm{Hc}$ & $\mathrm{H}$ & $\mathrm{N}$ & $\mathrm{X}$ & MS & - & - \\
\hline Agrostis hyemalis (Walter) Britton, Sterns \& Poggenb. & $\mathrm{Hc}$ & $\mathrm{H}$ & $\mathrm{N}$ & - & Am & - & - \\
\hline Aristida schiedeana Trin. \& Rupr. & $\mathrm{Hc}$ & $\mathrm{H}$ & $\mathrm{N}$ & $x$ & MS & - & - \\
\hline Aristida ternipes Cav. & $\mathrm{Hc}$ & $\mathrm{H}$ & $\mathrm{N}$ & $x$ & $\mathrm{Am}$ & - & - \\
\hline Briza subaristata Lam. & $\mathrm{Hc}$ & $\mathrm{H}$ & $\mathrm{N}$ & $X$ & MS & - & - \\
\hline Bromus carinatus Hook. \& Arn. & $\mathrm{Hc}$ & $\mathrm{H}$ & $\mathrm{N}$ & $x$ & Am & - & - \\
\hline Chloris submutica Kunth & $\mathrm{Hc}$ & $\mathrm{H}$ & $\mathrm{N}$ & $x$ & $\mathrm{Am}$ & - & - \\
\hline Digitaria ternata (Hochst. ex A. Rich.) Stapf & Tf & $\mathrm{H}$ & I & $X$ & $\mathrm{CO}$ & - & - \\
\hline Echinochloa oplismenoides (E. Fourn.) Hitchc. & $\mathrm{Hi}$ & $\mathrm{H}$ & $\mathrm{N}$ & - & MC & - & - \\
\hline Eragrostis intermedia Hitchc. var. intermedia & $\mathrm{Hc}$ & $\mathrm{H}$ & $\mathrm{N}$ & - & Am & - & - \\
\hline Hilaria belangeri (Steud.) Nash var. belangeri & $\mathrm{Hc}$ & $\mathrm{H}$ & $\mathrm{N}$ & $X$ & $\mathrm{Na}$ & - & - \\
\hline Leptochloa dubia (Kunth) Nees & $\mathrm{Hc}$ & $\mathrm{H}$ & $\mathrm{N}$ & $x$ & Am & - & - \\
\hline Lolium multiflorum Lam. & Tf & $\mathrm{H}$ & I & $x$ & $\mathrm{CO}$ & - & - \\
\hline Lycurus phleoides Kunth & $\mathrm{Hc}$ & $\mathrm{H}$ & $\mathrm{N}$ & - & $\mathrm{Na}$ & - & - \\
\hline Muhlenbergia dubia E. Fourn. & $\mathrm{Hc}$ & $\mathrm{H}$ & $\mathrm{N}$ & - & $\mathrm{Na}$ & - & - \\
\hline Muhlenbergia minutissima (Steud.) Swallen & Tf & $\mathrm{H}$ & $\mathrm{N}$ & $x$ & AM & - & - \\
\hline Muhlenbergia pubescens (Kunth) Hitchc. & $\mathrm{Hc}$ & $\mathrm{H}$ & $\mathrm{N}$ & - & $\mathrm{e}$ & M & - \\
\hline Muhlenbergia robusta (E. Fourn.) Hitchc. & $\mathrm{Hc}$ & $\mathrm{H}$ & $\mathrm{N}$ & - & MC & - & - \\
\hline Muhlenbergia virletii (E. Fourn.) Soderstr. & $\mathrm{Hc}$ & $\mathrm{H}$ & $\mathrm{N}$ & - & $\mathrm{e}$ & M & - \\
\hline Nassella mucronata (Kunth) R.W. Pohl & $\mathrm{Hc}$ & $\mathrm{H}$ & $\mathrm{N}$ & $X$ & MS & - & - \\
\hline Piptochaetium virescens (Kunth) Parodi & $\mathrm{Hc}$ & $\mathrm{H}$ & $\mathrm{N}$ & - & MC & - & - \\
\hline Poa annua L. & Tf & $\mathrm{H}$ & 1 & $X$ & $\mathrm{CO}$ & - & - \\
\hline Schizachyrium sanguineum (Retz.) Alston & $\mathrm{Hc}$ & $\mathrm{H}$ & $\mathrm{N}$ & $X$ & $\mathrm{CO}$ & - & - \\
\hline Sporobolus indicus (L.) R. Br. & $\mathrm{Hc}$ & $\mathrm{H}$ & $\mathrm{N}$ & $\mathrm{X}$ & Am & - & - \\
\hline Trisetum virletii E.Fourn. & $\mathrm{Hc}$ & $\mathrm{H}$ & $\mathrm{N}$ & - & $\mathrm{e}$ & M & - \\
\hline Zuloagaea bulbosa (Kunth) Bess & Gf & $\mathrm{H}$ & $\mathrm{N}$ & - & MS & - & - \\
\hline \multicolumn{8}{|l|}{ Pontederiaceae } \\
\hline $\begin{array}{l}\text { Heteranthera limosa (Sw.) Willd. } \\
\text { Smilacaceae }\end{array}$ & $\mathrm{Hi}$ & $\mathrm{H}$ & $\mathrm{N}$ & $x$ & AM & - & - \\
\hline $\begin{array}{l}\text { Smilax moranensis M. Martens \& Galeotti } \\
\text { Typhaceae }\end{array}$ & $\mathrm{Li}$ & $\mathrm{H}$ & $\mathrm{N}$ & - & e & $M$ & - \\
\hline Typha domingensis Pers. & $\mathrm{Hi}$ & $\mathrm{H}$ & $\mathrm{N}$ & $x$ & $\mathrm{CO}$ & - & - \\
\hline
\end{tabular}

